\title{
The auxin influx carrier, OsAUX3, regulates rice root development and responses to aluminium stress
}

\author{
Mei Wang ${ }^{1 *}$ | JiYue Qiao ${ }^{1 *} \mid$ ChenLiang $\mathrm{Yu}^{2 *} \mid$ Hao Chen ${ }^{1} \mid$ ChenDong Sun $^{1}$ | \\ LinZhou Huang ${ }^{3}$ | ChuanYou Li $^{3}$ | Markus Geisler ${ }^{4}$ | Qian Qian ${ }^{5}$ | De An Jiang ${ }^{1}$ | \\ YanHua Qi ${ }^{1}$ (D)
}

\author{
${ }^{1}$ State Key Laboratory of Plant Physiology and \\ Biochemistry, College of Life Sciences, \\ Zhejiang University, Hangzhou 310058, China \\ ${ }^{2}$ Vegetable Research Institute, Zhejiang \\ Academy of Agricultural Sciences, Hangzhou \\ 310021, China \\ ${ }^{3}$ State Key Laboratory of Plant Genomics, \\ National Center for Plant Gene Research, \\ Institute of Genetics and Developmental \\ Biology, Chinese Academy of Sciences, Beijing \\ 100101, China \\ ${ }^{4}$ Department of Biology, University of \\ Fribourg, Fribourg $\mathrm{CH}-1700$, Switzerland \\ ${ }^{5}$ State Key Laboratory of Rice Biology, China \\ National Rice Research Institute, Chinese \\ Academy of Agricultural Sciences, Hangzhou \\ 310006, China \\ Correspondence \\ YanHua Qi, State Key Laboratory of Plant \\ Physiology and Biochemistry, College of Life \\ Sciences, Zhejiang University, Hangzhou \\ 310058, China. \\ Email: qyhjp@zju.edu.cn \\ Funding information \\ National Basic Research Program of China \\ (973 Program), Grant/Award Number: \\ 2015CB942900; National Natural Science \\ Foundation of China, Grant/Award Number: \\ 31471460; State Key Laboratory of Plant \\ Genomics, Grant/Award Number: \\ 2017A0407-13; 111 project, Grant/Award \\ Number: B14027; Swiss National Funds, \\ Grant/Award Number: 31003A_165877
}

\begin{abstract}
In rice, there are five members of the auxin carrier AUXIN1/LIKE AUX1 family; however, the biological functions of the other four members besides OsAUX1 remain unknown. Here, by using CRISPR/Cas9, we constructed two independent OsAUX3 knock-down lines, osaux3-1 and osaux3-2, in wild-type rice, Hwayoung (WT/HY) and Dongjin (WT/DJ). osaux3-1 and osaux3-2 have shorter primary roots (PRs), decreased lateral root (LR) density, and longer root hairs (RHs) compared with their WT. OsAUX3 expression in PRs, LRs, and RHs further supports that OsAUX3 plays a critical role in the regulation of root development. OsAUX3 locates at the plasma membrane and functions as an auxin influx carrier affecting acropetal auxin transport. OsAUX3 is up-regulated in the root apex under aluminium (Al) stress, and osaux3-2 is insensitive to Al treatments. Furthermore, 1-naphthylacetic acid accented the sensitivity of WT/DJ and osaux3-2 to respond to Al stress. Auxin concentrations, Al contents, and Al-induced reactive oxygen species-mediated damage in osaux3-2 under Al stress are lower than in WT, indicating that OsAUX3 is involved in Al-induced inhibition of root growth. This study uncovers a novel pathway alleviating Al-induced oxidative damage by inhibition of acropetal auxin transport and provides a new option for engineering Al-tolerant rice species.
\end{abstract}

\section{KEYWORDS}

heavy metal stresses, lateral root initiation, polar auxin transport, primary root elongation, root hair development

\section{1 | INTRODUCTION}

Auxin is a critical plant hormone that regulates every aspect of plant growth and development (Kepinski, 2007; Ljung, 2013; Teale, Paponov, \& Palme, 2006). Auxin is produced particularly in shoot and root meristems and is transported over long distance in a nonpolar fashion in the vasculature to other parts of the plant. A second mode involves a cell-to-cell or polar auxin transport (PAT) employing

"These authors contributed equally to this work. carriers in the plasma membrane (Kramer \& Bennett, 2006). Auxin carriers include members of the AUXIN1/LIKE AUX1 (AUX1/LAX), PINFORMED, and ATP Binding Cassette B/P-glycoprotein families (Bennett et al., 1996; Cho, Lee, \& Cho, 2007; Geisler et al., 2005; Kerr \& Bennett, 2007; Murphy, Hoogner, Peer, \& Taiz, 2002; Noh, Murphy, \& Spalding, 2001; Petrásek et al., 2006; Swarup et al., 2008; Yang \& Murphy, 2009). In recent years, members of the PIN-LIKES family were also reported to be involved in auxin transport and homeostasis (Barbez \& Kleine-Vehn, 2013). PAT plays an important role in various aspects of plant growth and development, which is involved in 
regulation of embryogenesis, organogenesis, vascular tissue formation, lateral root (LR) initiation, and tropic responses (Friml \& Palme, 2002; Petrasek \& Friml, 2009; Peret et al., 2012; Swarup \& Bennett, 2003; Vieten, Sauer, Brewer, \& Friml, 2007).

In Arabidopsis, the AUX1/LAX family consists of four highly conserved members, AUX1, LAX1, LAX2, and LAX3 (Peret et al., 2012). AUX1 and LAX3 influence roots development, whereas LAX2 regulates vascular patterning in cotyledons (Bennett et al., 1996; Bhosale et al., 2018; Marchant et al., 2002; Peret et al., 2012; Swarup et al., 2001; Swarup et al., 2008; Vandenbussche et al., 2010). In tomato (Solanum lycopersicum), five AUX/LAX (SILAX1 to 5) genes revealed heterogeneous expression patterns, with tissue and developmental-stage specificity (Pattison \& Catala, 2012). In Medicago truncatula, MtLAX2, a paralogue of Arabidopsis AUX1, is required for nodule organogenesis (Roy et al., 2017). In Chinese cabbage (Brassica rapa L. ssp. pekinensis), it is suggested that $B r L A X$ genes may be involved in PAT during leafy head development (Gao et al., 2017). In rice, there are five members of the AUX1/LAX family (Shen et al., 2010). OsAUX1 was reported to function in regulation of LR development (Zhao et al., 2015). Previously, we have shown that OsAUX1, besides negatively regulating primary root (PR) elongation, positively regulates root hair (RH) development and responds to $\mathrm{Cd}$ stress (Yu et al., 2015). The roles of the other rice AUX1/LAX member remain unknown.

Aluminium (Al) rhizotoxicity is a major environmental stress that reduces crop production through inhibiting root elongation (Foy, 1988; Delhaize \& Ryan, 1995; Kochian, 1995; von Uexküll \& Mutert, 1995). Under heavy metal stresses, plants are known to dynamically regulate the transcription of auxin-related genes to adjust the effective accumulation of auxin within the plant for their survival (Wang, Wang, Zhao, Yang, \& Song, 2015). Several studies have demonstrated that Al-regulated inhibition of root growth may interact with auxin signalling. Auxin accumulation and distribution in roots was altered by the presence of Al (Kollmeier, Felle, \& Horst, 2000; Yang et al., 2014; Yang et al., 2017; Zhu et al., 2013). Auxin negatively mediates Al distribution in plant cells and $\mathrm{Al}$ tolerance by regulating ALS1 expression (Zhu et al., 2013). Auxin is a key signalling molecule that triggers an increase of malic acid against Al toxicity in wheat (Liu et al., 2017). Further, in the root apex transition zone of Arabidopsis, Al induces a localized enhancement of auxin signalling to regulate auxin biosynthesis (Yang et al., 2014). In OsPIN2 overexpression lines, it was found that the auxin efflux carrier alleviates Al-induced cell rigidity in rice root apex (Wu, Shen, Yokawa, \& Baluska, 2014). The expression of auxin transporter-like proteins and auxin efflux carrier components is significantly higher in Al-stressed alfalfa roots than in the control, whereas the expression of an auxin conjugate hydrolase is significantly lower (Zhou, Yang, Ren, Huang, \& An, 2014). However, the mechanism of Al-induced disruption of root PAT most likely resulting in inhibition of root growth remains unclear.

In this study, we decipher the roles of auxin influx carrier, OsAUX3, related to PR elongation, LR initiation, and RH development and in response to Al stress. OsAUX3 transcription was induced by $\mathrm{Al}$ treatment, and the osaux 3 mutant was insensitive to Al stress, indicating that OsAUX3 functions as a negative regulator decreasing rice $\mathrm{Al}$ tolerance in roots. Knowledge of this molecular mechanism may contribute to the breeding of Al-tolerant crops.

\section{2 | METHODS AND MATERIALS}

\section{1 | Plant materials and growth conditions}

Japonica wild-type rice Hwayoung (WT/HY) and Dongjin (WT/DJ), OsAUX3-related transgenic mutants, and OsAUX3 overexpression lines were planted in nutrient solution as previously described (Wang et al., 2014; Xu et al., 2014). Phytohormone treatment was performed with $1 \mu \mathrm{M}$ of indole-3-acetic acid (IAA), or $0.01 \mu \mathrm{M}$ of 2,4dichlorophenoxyacetic acid (2,4-D) and $0.01 \mu \mathrm{M}$ of 1-naphthylacetic acid (NAA) for 7 days. Al treatment was performed in a $0.5-\mathrm{mM} \mathrm{CaCl}_{2}$ solution ( $\mathrm{pH} 4.5$ ) with different concentration of $\mathrm{AlCl}_{3}$ as indicated in the figure legends.

\subsection{Construction and identification of osaux3 mutants}

The CRISPR/Cas9 system was used to establish OsAUX3 knock-down lines (Xie, Minkenberg, \& Yang, 2015). WT/HY and WT/DJ were infected by the Agrobacterium strain EHA105 transformed with OsAUX3-pRGEB32 as previously described (Hiei, Ohta, Komari, \& Kumashiro, 1994). The related editing site was found through DNA sequencing. The design of specific primers was based on editing sites to identify the original generation (TO) of transgenic rice. Seeds of selffertilized T0 were harvested as transgenic line (T1). Homozygous osaux3 mutants were identified with Cas9 specific primers and OsAUX3 specific primers. Cas9 label was removed from the homozygous osaux3 mutants. Homozygous T2 seeds were used throughout this study. The primers used for plasmid construction are listed in Table S1.

\subsection{Construction and transformation of binary vectors}

The ORF of OsAUX3 (Os05g37470) was amplified from the full-length CDNA of WT/DJ using the primers listed in Table $\mathrm{S} 1$ and cloned into pCAMBIA1300-sGFP to create the 35s:OsAUX3-sGFP fusion construct. For constructing the ProOsAUX3:OsAUX3-sGFP and ProOsAUX3:GUS (pBI101.3), $2.3 \mathrm{~kb}$ of the OsAUX3 promoter was used to replace the CaMV35S promoter. These vectors were introduced into Agrobacterium strain EHA105 using electroporation and transformed into WT/DJ using the callus infection method as described previously (Hiei et al., 1994).

\subsection{Subcellular localization of OsAUX3}

35s:OsAUX3-sGFP and ProOsAUX3:OsAUX3-sGFP fusion constructs were transiently expressed in tobacco epidermal cells by agrobacterium-mediated transformation as previously described (Qi et al., 2012). The two constructs were also polyethylene glycolcalcium transfected into rice protoplasts, which were prepared from stems of 10-day-old rice seedlings. Images were acquired using the two-photon microscope, Zeiss LSM710 (Carl Zeiss, Oberkochen, Germany). 


\section{5 | $\beta$-Galactosidase staining and analysis of $\beta$-galactosidase activity}

The maximum auxin response reporter, DR5:GUS, was introduced into Agrobacterium strain EHA105 and was transformed into WT/HY and osaux3-1 mutants. $\beta$-Galactosidase (GUS) staining was performed as described previously (Jefferson, Kavanagh, \& Bevan, 1987). Root tissues were vacuumed-infiltrated in staining solution for $20 \mathrm{~min}$ and incubated at $37^{\circ} \mathrm{C}$. After staining, tissues were soaked in $70 \%$ ethanol to remove chlorophyll and surface dyes and observed by using Nikon AZ100 microscope (Nikon Corporation, http://www. nikoninstruments.com.cn/index.html). For quantification of GUS activity, $50 \mathrm{mg}$ of root tissues were grounded in liquid nitrogen, resuspended in phosphate-buffered saline (PBS) solution $(\mathrm{pH} 7.4)$, and centrifuged at $4200 \mathrm{~g}$ for $10 \mathrm{~min}$, and the supernatant was collected. GUS activity was measured by NanoQuant infinite M200 pro (www. eastwin.com.cn) spectrophotometrically at a wavelength of $450 \mathrm{~nm}$ using the Plant GUS ELISA Kit (www.bangyi-sh.com).

\section{6 | EdU staining}

One centimetre of root tips of 3-day-old seedlings was treated with 50- $\mathrm{M}$ 5-ethynyl-2'-deoxyuridine (EdU) of culture medium for 1$2 \mathrm{hr}$ as described in EdU Flow Cytometry Assay Kits (http://www. ribobio.com/sitecn/Products.aspx?id=37) and observed under a LSM710 NLO microscope (Zeiss, http://www.zeiss.com/corporate/ en_de/home.html).

\section{7 | TTC staining}

PRs of 3- and 5-day-old seedlings were incubated in $0.4 \% 2,3,5$ triphenyltetrazolium chloride (TTC) solution for $3 \mathrm{hr}$, vacuum-treated for $20 \mathrm{~min}$, washed three times with $\mathrm{dd}_{2} \mathrm{O}$, and dissociated in $10 \%$ hydrochloric acid for $10 \mathrm{~min}$. Staining was observed under a Nikon SMZ 745T microscope (Nikon Corporation, http://www. nikoninstruments.com.cn/index.html) using $10 \%$ glycerin as transparent reagent.

\section{8 | Measurement of IAA concentration and IAA transport}

Free IAA concentrations in 1-cm sections of WT/HY and osaux3-1 mutant root tips were measured by NanoQuant infinite M200 pro (www.eastwin.com.cn) using the plant IAA ELISA Kit (www.bangyish.com). For that, $50 \mathrm{mg}$ of root samples were grounded in liquid nitrogen, resuspended in PBS solution ( $\mathrm{pH}$ 7.4), and centrifuged at $4200 \mathrm{~g}$ for $10 \mathrm{~min}$. The supernatant was used to measure IAA concentrations spectrophotometrically at $450 \mathrm{~nm}$. For the analyses of polar ${ }^{3} \mathrm{H}$-IAA transport in rice roots, $1 \mathrm{~cm}$ of root tip was performed using a 1450 MicroBeta TriLux liquid scintillation counter (PerkinElmer, http:// www.perkinelmer.com/) as described previously (Qi et al., 2008). IAA export from rice protoplasts was performed as described in $\mathrm{Yu}$ et al. (2015).

\section{9 | Al quantification and Morin staining}

For a quantification of $\mathrm{Al}$ concentrations in root tips, $1 \mathrm{~cm}$ of root tips treated with $25-\mu \mathrm{M} \mathrm{AlCl}_{3}$ was excised, washed six times with 0.2-mM $\mathrm{CaCl}_{2}$, and incubated in $2-\mathrm{M} \mathrm{HCl}$ for $48 \mathrm{hr}$, and supernatants were then analysed by ICP-OES (Optical Emission Spectrometer, Optima8000, PerkinElmer). For Morin staining, 1-cm apical root cuts was washed for $5 \mathrm{~min}$ with $0.2-\mathrm{mM} \mathrm{CaCl}_{2}$ and sliced into $100-\mu \mathrm{m} \mathrm{sec}$ tion by using a vibratome (Leica VT1000 S). Slices were Morin stained for $5 \mathrm{~min}$ and washed twice with $0.2-\mathrm{mM} \mathrm{CaCl}_{2}$, and fluorescence intensity was observed under a LSM710 NLO Zeiss microscope (Zeiss, http://www.zeiss.com/corporate/en_de/home.html).

\subsection{0 | Analyses of root $\mathrm{H}_{2} \mathrm{O}_{2}$ and CAT concentrations}

Analysis of $\mathrm{H}_{2} \mathrm{O}_{2}$ accumulation in the root tips was performed by using $2^{\prime}, 7^{\prime}$-dichlorofluorescin diacetate (H2DCF-DA). One-centimetre root tips were cut and incubated with $50-\mu \mathrm{M}$ H2DCF-DA solution for 10 min under vacuum and washed two times with $0.2-\mathrm{mM} \mathrm{CaCl}_{2}$. Root tips were then observed under a LSM710 NLO Zeiss microscope (Zeiss, http://www.zeiss.com/corporate/en_de/home.html). For measurement of $\mathrm{H}_{2} \mathrm{O}_{2}$ and CAT concentration in root tips, 1-cm root tips of treated seedlings were grounded in liquid nitrogen, resuspended in PBS ( $\mathrm{pH}$ 7.4), and centrifuged at $4200 \mathrm{~g}$ for $10 \mathrm{~min}$. Collected supernatants were used to measure $\mathrm{H}_{2} \mathrm{O}_{2}$ and CAT concentration by NanoQuant infinite M200 pro (www.eastwin.com.cn) spectrophotometrically at a wavelength of $450 \mathrm{~nm}$ by using the Plant $\mathrm{H}_{2} \mathrm{O}_{2}$ ELISA and Plant CAT ELISA Kits (www.bangyi-sh.com), respectively.

\subsection{1 | RNA extraction and quantitative RT-PCR}

Total RNA was extracted from tissues after various treatments using a commercial kit and according to the manufacturer's instructions (Tiangen, Hangzhou, China http://www.tiangen.com/). Quantitative RT-PCR (qRT-PCR) was performed as described previously (Wang et al., 2010; Wang et al., 2014); OsACTIN (Os03g50885) and OsUBI (Os03g13170) were used as internal control for qRT-PCR. Primers used are listed in Table S2.

\section{3 | RESULTS}

\section{1 | Knock-down mutants of OsAUX3 are insensitive to auxin, and OsAUX3 expression is induced by auxin in PR}

In a previous study, we reported that OsAUX1 plays an important role in root development and in responses to Cd stress (Yu et al., 2015). To clarify the biological function of OsAUX3, the closest homology of OsAUX1 (Figures S1 and S2), we constructed two independent alleles of osaux3 mutants using CRISPR/Cas9 technology, and OsAUX3 overexpression lines in the WTs, HY and DJ (Figures S3 and S4). These homozygous T2 lines were tested under hydroponic culture condition for 7 days. Our 
results show that the PR length of osaux3-1 or osaux3-2 mutants was 25$30 \%$ shorter than their corresponding WT, whereas the PR length of OsAUX3 overexpression lines, 35S:OsAUX3-3 and 35s:OsAUX3-6, was $35 \%$ longer than WT/DJ (Figure 1a,b). Importantly, the PR length of osaux3-complemented lines was close to their WT, indicating that the PR alteration in osaux 3 or OsAUX3 overexpression lines was caused by altered OsAUX3 expression. The PR of the osaux3-2 mutant was not significantly reduced in comparison with WT/DJ in the presence of IAA or the synthetic auxins, 2,4-D or NAA, respectively (Figure 1c), suggesting that osaux3-2 is insensitive to auxin. Interestingly, OsAUX3 expression was highly induced by IAA, 2,4-D, and NAA, especially in the PR apex, as demonstrated by qRT-PCR and GUS staining (Figure 1d,e). This together suggests that OsAUX3 might participate in regulation of auxinmediated PR development.
To deeper understand the cause of alteration of PR length in osaux3, EdU staining of the PR apex revealed reduced fluorescence intensities in osaux3-1 and osaux3-2 compared with WT/HY and WT/DJ, whereas fluorescence was higher in both OsAUX3 overexpression lines, 35s:OsAUX3-3 and 35s:OsAUX3-6, respectively. These results indicate that the cell division activity is dependent on OsAUX3, leading likely to a short-root phenotype in the mutants.

\section{2 | Deferred LR initiation and decreased expression in genes related to LR initiation and development in osaux3-2}

To better understand OsAUX3 function during root development, the density of LRs in osaux3-2 and 35S:OsAUX3-3 was measured
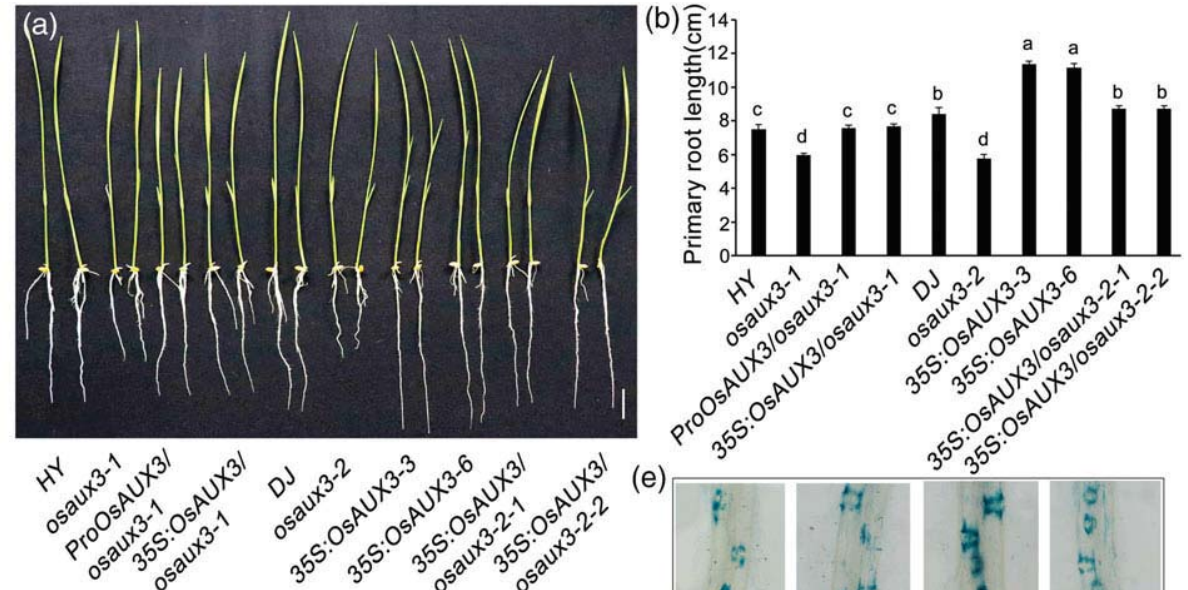

(c)

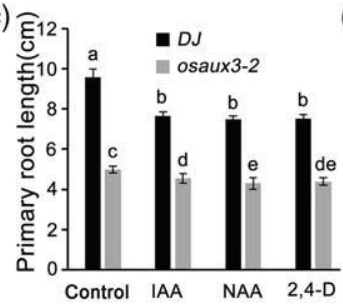

(d)

(f)
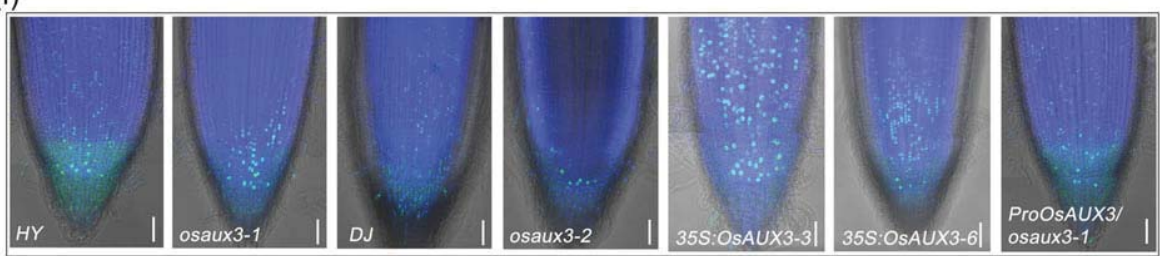

FIGURE 1 Phenotypic characterizing of OsAUX3 gain and loss-of-function phenotypes. (a) Phenotypes of wild-type rice Hwayoung (WT/HY), osaux3-1, its complemented transgenic lines, ProOsAUX3/osaux3-1 and 35S:OsAUX3/osaux3-1; wild-type rice Dongjin (WT/DJ), osaux3-2,

OsAUX3 overexpression lines, 35S:OsAUX3-3 and 35s:OsAUX3-6, and complemented transgenic lines, 35s:OsAUX3-2-1 and 35S:OsAUX3-2-2 for 7-day-old seedling, from left to right. Bar $=2 \mathrm{~cm}$. (b) Quantification of primary root (PR) length of mentioned lines in (a). Significant difference to WT are indicated by letters, $n=3$ with 10 seedlings each (Duncan's test, $P<0.05$ ). (c) Quantification of PR length of WT/DJ and osaux3-2 7 days (days after germination) under grown on solvent, $1 \mu \mathrm{M}$ of indole-3-acetic acid (IAA), $0.01 \mu \mathrm{M}$ of 1-naphthylacetic acid (NAA), or 0.01- $\mu$ M 2,4dichlorophenoxyacetic acid (2,4-D) treatments. Significant difference to WT are indicated by letters, $n=3$ with 10 seedlings each (Duncan's test, $P<0.05$ ). (d) OsAUX3 expression in PR apex of WT/DJ for 3-day-old seedling under normal condition, $10 \mu M$ of IAA, $0.1 \mu M$ of NAA, and $0.1 \mu M$ of 2,4-D treatments. qRT-PCR experiments were analysed using three independent biological replicas. OsACTIN and OsUBI were used as internal controls. Asterisks indicate significant differences compared with solvent control ( ${ }^{* *} P<0.01$; ${ }^{*} P<0.05$; $t$ test). (e) GUS staining of PRs of ProOsAUX3:GUS lines for 3-day-old seedling under normal condition, $10 \mu \mathrm{M}$ of IAA, $0.1 \mu \mathrm{M}$ of NAA, and $0.1 \mu \mathrm{M}$ of 2,4-D treatments. In each treatment, 10 seedlings were used. Bar $=500 \mu \mathrm{m}$. (f) Ethynyl-2'-deoxyuridine staining for PR apex in WT, osaux3 mutants, OsAUX3 overexpression lines and complemented transgenic lines for 3-day-old seedlings. In each treatment, 10 seedlings were used. Bar $=50 \mu \mathrm{m}$ 
(Figure 2a,b). LR initiation in osaux3-2 was delayed in 3-day-old seedlings, and the density of LRs in osaux3-2 was significantly decreased in 5- or 7-day-old seedlings. To get further insight into the basic reason for the reduced density of LRs in osaux3-2, LR primordia were observed by TTC staining (Figure 2c). In 3- and 5day-old PRs, the number of LR primordia was significantly decreased in osaux3-2 but increased in 35S:OsAUX3-3 (Figure 2c). These results indicate that knock-down of OSAUX3 influences LR initiation. Both histochemical GUS staining in LRs of ProOsAUX3:GUS and fluorescence quantification in LRs of ProOsAUX3:OsAUX3-GFP showed that OsAUX3 is expressed in LR primordia and extended LRs, further suggesting that OsAUX3 function is also involved in regulating LR initiation (Figure 2d).

Previously, auxin-induced LR initiation by the regulation of cell cycle-related gene expression was reported (Guo, Song, Wang, \& Zhang, 2007). About 90 putative core cell cycle genes were reported to participate in cell division. They belong to cyclin-dependent kinases (CDK), E2F transcription factors, CDK inhibitors, CDK subunit proteins, homologues of the retinoblastoma protein and the protein kinase Wee families (Guo et al., 2007). Moreover, it was shown that cyclins and CDKs affect LR densities and that CYCDs regulate the
G1-S transition to promote LR initiation (Nieuwland et al., 2009; Sanz et al. 2011). Especially, CYCD4;1 controls cell length in the pericycle of the basal meristem and affects the formation of LR (Nieuwland et al., 2009). Further, CDK is involved in LR induction in response to auxin by regulating the levels of interactor of CDK/Kip-Related Protein 2 (Verkest et al., 2005; Sanz et al., 2011). Our results show that the cell cycle-related genes OsCYCD4;1, OsCYCB2;2, OsCYCU4;3, OsCDKC;1, OsCDKC;3, and OsCKL:10 were dramatically reduced in osaux3-2 compared with WT/DJ but increased in 35S:OsAUX3-3, which suggests that OsAUX3 might also function in regulating LR initiation through mediating expressions of those cell cycle-related genes (Figure 2e).

\subsection{Increased RH length in osaux3-2 and OsAUX3 expression in $\mathrm{RH}$ cell}

In our previous research, we have shown that OsAUX1 expression is different in comparison with its paralogous gene, AtAUX1, which is not expressed in $\mathrm{RH}$ s but still able to regulate $\mathrm{RH}$ development (Jones et al., 2009; Yu et al., 2015). We wondered whether OsAUX3
FIGURE 2 Analysis of OsAUX3 in lateral root (LR) development. (a) LR growth of wildtype rice Dongjin (WT/DJ), osaux3-2, and 35S: OsAUX3-3 seedlings at 3, 5, and 7 days. Bar $=2 \mathrm{~cm}$. (b) Quantification of LR density in WT/DJ, osaux3-2, and 35S:OsAUX3-3, respectively. Ten biological replicas were measured. Columns with different letters indicate significant differences to WT (Duncan's test, $P<0.05$ ). (c) 2,3,5triphenyltetrazolium chloride staining in primary root of WT/DJ, osaux3-2, and 35S: OsAUX3-3 seedlings. Left, $0.5-\mathrm{cm}$ samples without LR were taken from the root-stem base in the above three seedlings of 3-day-old of primary root; right, 1-cm samples without LR were taken from $4 \mathrm{~cm}$ of root-stem base in WT/DJ, $3 \mathrm{~cm}$ of stem base in osaux $3-2$, and $5 \mathrm{~cm}$ of root-stem base in 35S:OsAUX3-3. Bar $=1 \mathrm{~mm}$. (d) Expression pattern of OsAUX3 in LR. GUS staining in LR of 5-dayold ProOsAUX3:GUS and fluorescence imaging of LR of 5-day-old ProOsAUX3:OsAUX3-GFP seedlings. Upper panels from left to right show lateral root primordia and initiated LR, respectively, whereas lower panels from left to right indicate the transverse section and longitudinal section of the mature LR, respectively. Bar $=100 \mu \mathrm{m}$. (e) Relative mRNA levels of genes related to LR initiation in WT/ DJ, osaux3-2 and 35S:OsAUX3-3. qRT-PCR experiments were analysed using three independent biological replicas. OSACTIN and OsUBI were used as internal controls. Asterisks indicate significant differences compared with WT/DJ, respectively $\left({ }^{* *} P<0.01 ;{ }^{*} P<0.05 ; t\right.$ test) [Colour figure can be viewed at wileyonlinelibrary.com]
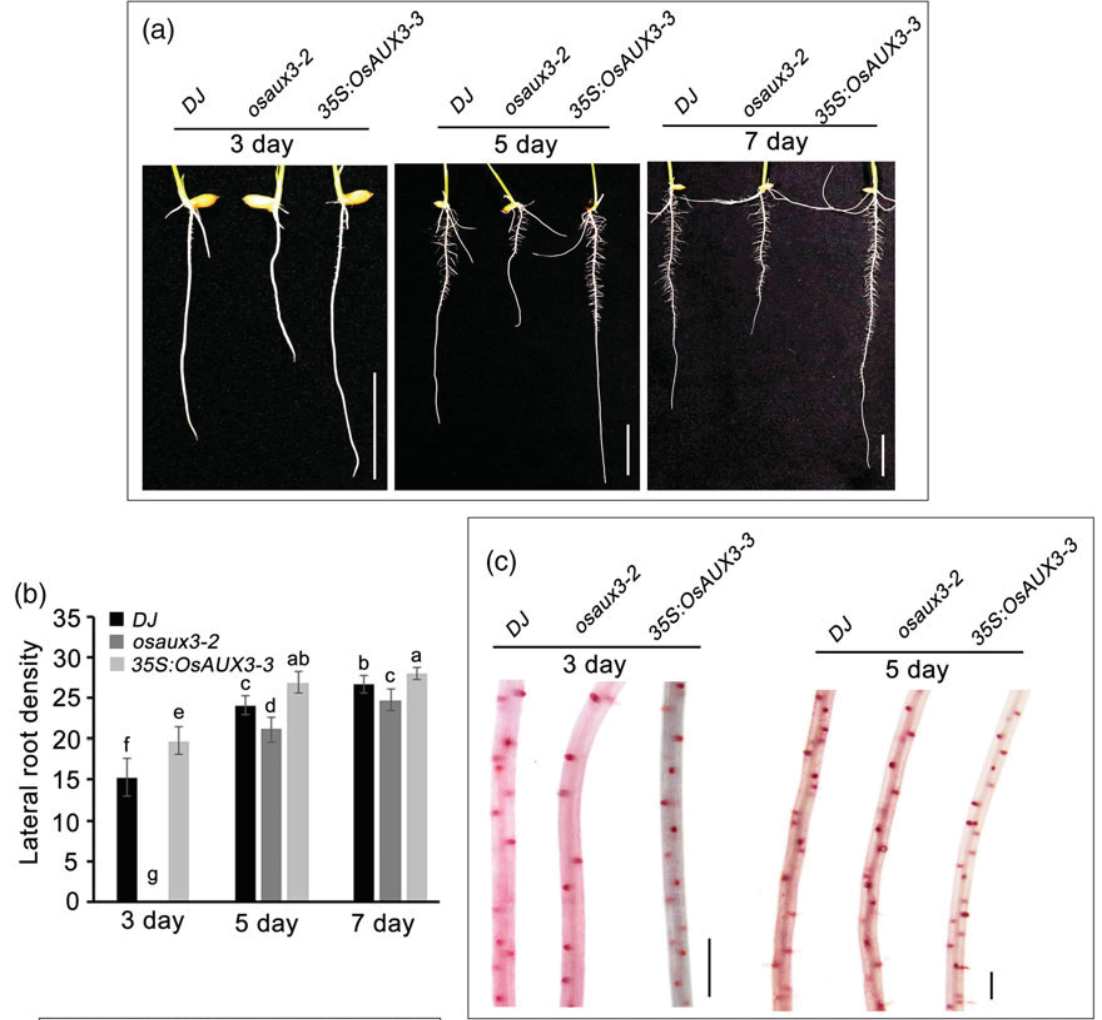

(d)

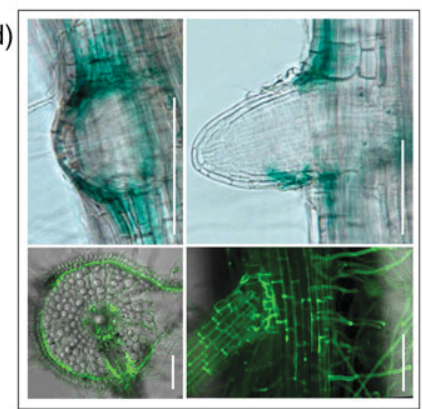


would be also involved in $\mathrm{RH}$ growth regulation and expressed in $\mathrm{RH}$, indicating that their molecular mechanisms would be conserved between rice and Arabidopsis. RH length in WT and osaux3-2 in 3day-old seedlings were found to be increased by $60 \%$ compared with the WT (Figure 3a,b), which is different to the osaux1 mutant showing shorter $\mathrm{RH}$.

The expression pattern of OsAUX3 was investigated using transgenic rice expressing ProOsAUX3:OsAUX3-GFP (Figure 3c). The analysis revealed that OsAUX3 is expressed in each period $\mathrm{RH}$ including young RHs, developing $\mathrm{RHs}$, and mature $\mathrm{RHs}$. This is not the case for OsAUX1, which is not expressed in mature RHs, indicating a functional difference in regulating $\mathrm{RH}$ development not only in between monocot and dicot plants but also in close rice homologies.

\subsection{OsAUX3 contributes to an acropetal IAA translocation by functioning as an importer}

Members of the AUX1/LAX family in Arabidopsis function as auxin influx carriers during auxin distribution. To deeper understand the biological function of OsAUX3, we further investigated its subcellular localization and its auxin transport capacity in osaux 3 mutants. By using the 35S:OsAUX3-GFP constructs, OsAUX3 was co-localized with the plasma membrane marker, pm-rbCD3-1008, in tobacco and rice epidermal cells and protoplasts, respectively (Figure 4a), implying that OsAUX3 and AtAUX1 might share overlapping auxin transport functionalities. In agreement, both osaux 3 alleles showed significantly enhanced IAA export from rice protoplasts prepared from osaux3-1 and osaux3-2 plants, in comparison with their corresponding WT (Figure 4b). Increased export caused by loss of function of a plasma membrane transporter can only be explained by an import directionality. In this scenario, a lack of reimport of effluxed radiolabeled IAA results in elevated net export compared with the WT. As a result, acropetal auxin transport in osaux3-1 and osaux3-2 roots is decreased drastically, in comparison with the WT (Figure 4c,d). These results suggest that OsAUX3 functions as an auxin importer involved in acropetal auxin transport.

\subsection{Al stress induces OsAUX3 expression in the rice root, and NAA increases the inhibition of PR growth under $\mathrm{Al}$ stress}

Al toxicity inhibiting root elongation is a major limiting factor for rice growth. It was found that Al-regulated inhibition of root growth is regulated by auxin biosynthesis and signalling. In order to uncover if OsAUX3-mediated auxin transport is involved in responses to $\mathrm{Al}$ stress, we first quantified OsAUX3 expression in 3-day-old WT/DJ seedling under control conditions and Al treatment by qRT-PCR and GUS staining in the PR of ProOsAUX3:GUS plants (Yang et al., 2014). The results show that OsAUX3 expression was significantly increased by $\mathrm{AlCl}_{3}$ treatment for $3 \mathrm{hr}$ (Figure 5a,b), suggesting that OsAUX3 might function in responses to Al stress.

To better understand the molecular mechanisms of OsAUX3 responses to Al stress, the morphology of WT/DJ, osaux3-2, and 35S:OsAUX3-3 in 7-day-old seedlings was observed under various concentrations of $\mathrm{Al}$ treatment. It was found that PR growth of WT/DJ, osaux3-2, and 35S:OsAUX3-3 was inhibited to a different degree by increasing Al concentrations (Figure $5 c$,d). However, compared with WT/DJ, PR growth in osaux3-2 was insensitive to Al stress, suggesting that decreased acropetal auxin transport in osaux3-2 might affect the sensitivity to Al stress. Interestingly, the inhibition of PR elongation by $\mathrm{Al}$ treatment was enhanced by addition of the synthetic auxin, NAA (Figure $5 \mathrm{e}, \mathrm{f}$ ). Similarly, root growth inhibition under different concentrations of $\mathrm{Al}$ stress for $24 \mathrm{hr}$ was also significantly alleviated in osaux3-2 compared with the WT/DJ (Figure 5g), and after $24 \mathrm{hr}$ of NAA exposure, the inhibition of PR elongation under Al stress (a)

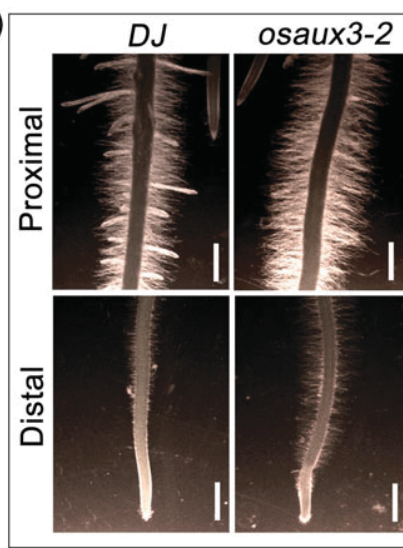

(b)

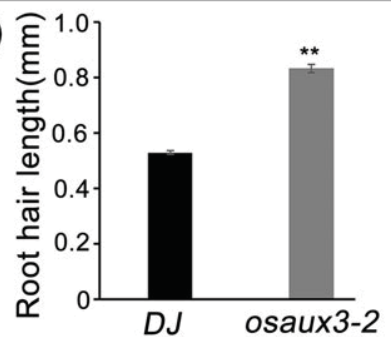

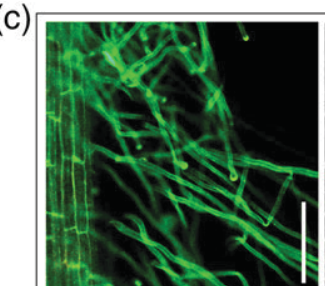
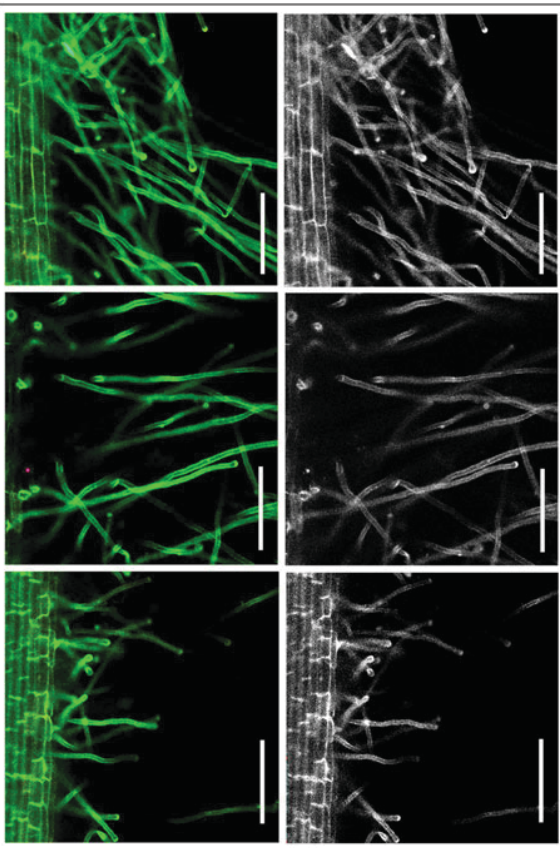

FIGURE 3 Morphology of root hair (RH) in wild-type rice Dongjin (WT/DJ) and osaux3 mutant and OsAUX3 expression in RH. (a) Comparative analysis of $\mathrm{RH}$ phenotype in WT/DJ and osaux3-2 for 3-day-old seedlings. Bar $=1 \mathrm{~mm}$. (b) Statistical analysis of RH length of WT/DJ and osaux3-2 for 3-day-old seedlings. Ten biological replicas were measured for this test. Asterisks indicate significant differences compared with WT/DJ $\left({ }^{* *} P<0.01 ; t\right.$ test). (c) OsAUX3 expression in the $\mathrm{RH}$. Fluorescence microscopy of RH of 3day-old ProOsAUX3:OsAUX3-GFP seedlings. Left, GFP channel (green represents GFP signals; yellow represents auto-fluorescence of roots); right, bright-field images. Upper to lower panels show mature $\mathrm{RHs}$ (length $\approx 800 \mu \mathrm{m}$ ), developing RHs (length $\approx 250 \mu \mathrm{m}$ ) and young RHs (length $\approx 100 \mu \mathrm{m}$ ),

respectively. Bar $=100 \mu \mathrm{m}$ [Colour figure can be viewed at wileyonlinelibrary.com] 
(a)
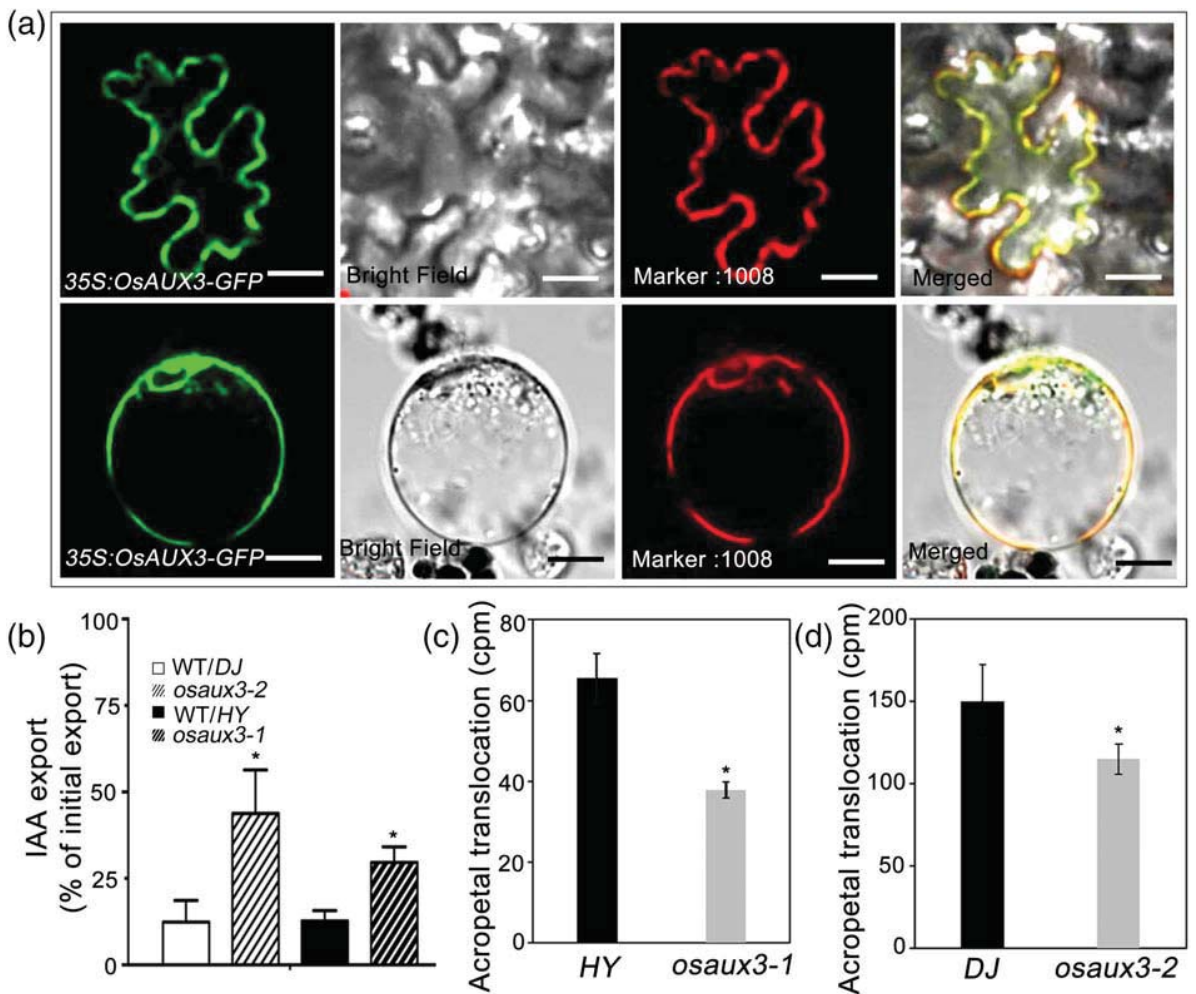

FIGURE 4 Subcellular localization of OsAUX3 and auxin transport in wild-type (WT) and osaux3 mutants. (a) 35S:OsAUX3-GFP fusion construct transiently expressed in tobacco and rice. Co-transformation of 35S:OsAUX3-GFP with plasma membrane marker pm-rbCD3-1008. Left to right: green fluorescence of OsAUX3-GFP, bright-field images, red fluorescence of the protoplast membrane marker pm-rb CD3-1008, and merged microscope images. Bar $=50 \mu \mathrm{m}$. (b) Indole-3-acetic acid (IAA) export from osaux3-2 and osaux3-1 and from corresponding WT protoplasts, WT rice Dongjin (WT/DJ) and Hwayoung WT/HY, respectively, after 20 min. Significant differences (unpaired $t$ test with Welch's correction, $P<0.05$ ) to WT controls are indicated by an asterisk (mean $\pm S E ; n \geq 3$ ). Analysis of acropetal IAA transport in primary root of 3-day-old (c) WT/DJ and osaux3-2. (d) WT/HY and osaux3-1 seedling. These experiments were performed using five independent biological replicas. Asterisk indicate significant differences $\left({ }^{*} P<0.05 ; t\right.$ test) [Colour figure can be viewed at wileyonlinelibrary.com]

was enhanced (Figure 5h). According to a previous study, decreased auxin synthesis results in $\mathrm{Al}$ insensitivity in Arabidopsis (Yang et al., 2014). These findings together with our experiments imply that the sensitivity to Al stress might depend on local changes in auxin concentrations caused by reduced auxin transport in osaux3-2.

\section{6 | Reduced auxin concentration in osaux3-2 might confer Al stress insensitivity to osaux3-2}

To confirm the effect of auxin on Al sensitivity, we further analysed the auxin distribution in WT and osaux3-1 lines using the auxin response marker, DR5:GUS, transformed into WT/HY and osaux3-1 (Figure 6a). DR5:GUS staining and DR5-GUS activity were significantly reduced in osaux3-1 compared with WT/HY (Figure 6b). In agreement, also, the content of auxin in the root tip of osaux3-2 mutant was reduced compared with the WT control (Figure 6c). Interestingly, the auxin concentration in both WT/DJ, osaux3-2, and 35S:OsAUX3-3 was increased under Al stress; however, the increase of auxin in osaux3-2 was less pronounced than in WT/DJ, suggesting that an inhibition of acropetal auxin translocation in osaux3-2 (Figure 4d) might be responsible for this event. These results with those from Figure 5 suggest that reduced auxin levels might be the primary cause for a reduced root sensitivity of osaux3-2 in response to Al.

Furthermore, we wondered whether decreased Al sensitivity also affected the Al content of osaux3-2 PR tips. Al contents of WT/DJ, osaux3-2, and 35S:OsAUX3-3 PR tips of 3-day-old seedlings exposed to $25-\mu \mathrm{M} \mathrm{AlCl} 3$ for $3 \mathrm{hr}$ were analysed by ICP and Morin fluorescence staining (Figure 6d,e). ICP analyses revealed that $\mathrm{Al}$ contents in osaux3-2 PR apex were reduced by $60 \%$ but found to be increased by $25 \%$ in $35 \mathrm{~S}:$ OSAUX3-3 compared with the WT/DJ, respectively. Further, the distribution and accumulation of $\mathrm{Al}$ in the PR was visualized using the fluorochrome Morin (Del et al., 1990; Mile et al., 2015). Root sections of PRs indicate that Morin fluorescence intensities were significantly lower in osaux3-2 but higher in 35s:OsAUX3-3 compared with the WT/DJ. Both experiments demonstrate that Al accumulation in the PR apex of osaux3-2 is reduced.

\section{7 | Oxidative damage in osaux3-2 is reduced under Al stress}

Accumulation of reactive oxygen species (ROS) under Al stress has been widely reported (Yamamoto, Kobayashi, Devi, Rikiishi, \& Matsumoto, 2002), and the concentration of $\mathrm{H}_{2} \mathrm{O}_{2}$ is often used as an indicator of oxidative damage. Root tips are susceptible to 
(a)

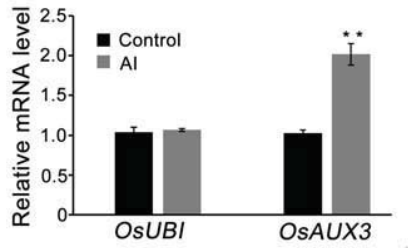

(c)
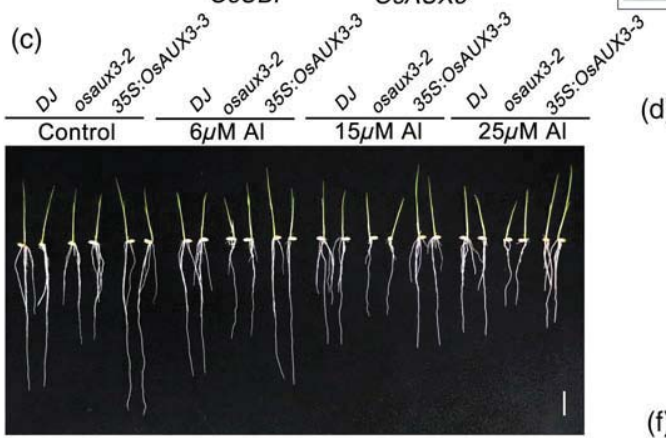

(e)

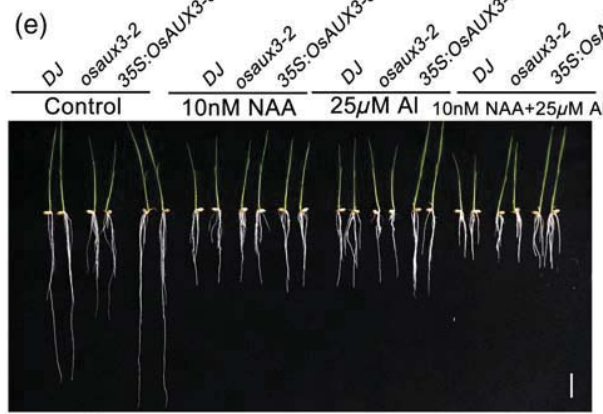

(g)

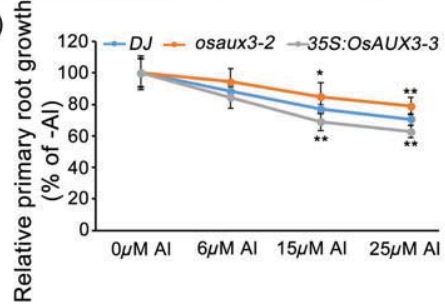

(b)

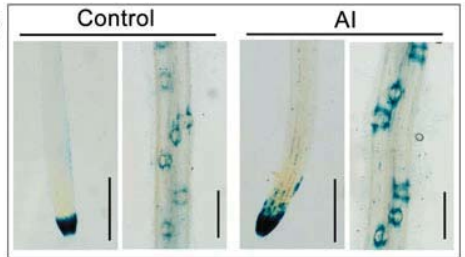

(d)

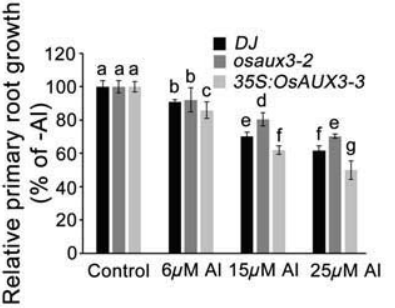

(f) $\frac{5}{5}$

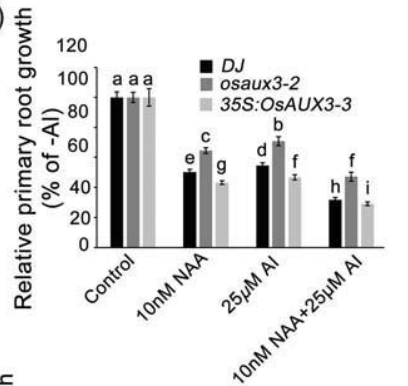

(h) $\frac{5}{3}$

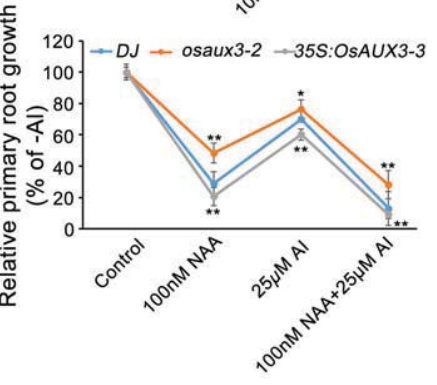

FIGURE 5 OsAUX3 expression level and primary root $(\mathrm{PR})$ growth in wild-type rice Dongjin WT/DJ, osaux3-2, and 35S:OsAUX3-3 under aluminium (Al) treatment. (a) Relative mRNA level of OsAUX3 in the PR apex of 3day-old WT/DJ seedling under control and Al treatment. qRT-PCR experiments were analysed using three independent biological replicas. OsACTIN and OsUBI were used as internal controls. Asterisks indicate significant differences (** $P<0.01 ; t$ test). (b) $\beta$ -

Galactosidase staining in the PR of 3-day-old ProOsAUX3:GUS seedlings with and without Al treatment. For each treatment $(n=3), 10$ seedlings were used for this test.

Bar $=500 \mu \mathrm{m}$. (c) Phenotypic characterization of WT/DJ, osaux3-2, and 35S:OsAUX3-3 under 0-, 6-, 15-, or 25- $\mu \mathrm{M} \mathrm{AlCl}_{3}$ treatment for 7 days. Bar $=2 \mathrm{~cm}$. (d) Analysis of relative PR growth of WT/DJ, osaux3-2, and 35S: OsAUX3-3 under 0-, 6-, 15-, or 25- $\mu \mathrm{M} \mathrm{AlCl}_{3}$ treatment for 7 days. Ten seedlings of each line were used here, $n=3$. Columns with different letters indicate significant differences (Duncan's test, $P<0.05$ ). (e) Phenotypic characterization of WT/DJ, osaux3-2, and 35S:OsAUX3-3 under 0, 10-nM 1-naphthylacetic acid (NAA), 25- $\mu \mathrm{M} \mathrm{AlCl}$, or 10-nM NAA + 25- $\mu \mathrm{M} \mathrm{AlCl} 3$ treatments for 7 days. $B a r=2 \mathrm{~cm}$. (f) Analysis of relative PR growth of WT/DJ, osaux3-2, and 35S: OsAUX3-3 under 0, 10-nM NAA, 25- $\mu$ M $\mathrm{AlCl}_{3}$, or $10-n M \mathrm{NAA}+25-\mu \mathrm{M} \mathrm{AlCl}{ }_{3}$ treatments for 7 days. Ten seedlings of each line were used here, $n=3$. Columns with different letters indicate significant differences (Duncan's test, $P<0.05$ ). (g) Analysis of relative PR growth in WT/DJ, osaux3-2, and 35S:OsAUX3-3 under 0-, 6$15-$, or $25-\mu \mathrm{M} \mathrm{AlCl}_{3}$ treatment for $24 \mathrm{hr}$. Ten seedlings of each line were used here, $n=3$. Asterisks indicate significant differences compared with WT/DJ, respectively $\left({ }^{* *} P<0.01 ;{ }^{*} P<0.05 ; t\right.$ test). (H) Analysis of relative PR growth of WT/DJ, osaux3-2, and 35S:OsAUX3-3 under 0, 100-nM NAA, 25- $\mu$ M $\mathrm{AlCl}_{3}$, or $100-n M \mathrm{NAA}+25-\mu \mathrm{M} \mathrm{AlCl} 3$ treatments for $24 \mathrm{hr}$. Ten seedlings of each line were used here, $n=3$. Asterisks indicate significant differences compared with WT/DJ, respectively $\left({ }^{* *} P<0.01 ;{ }^{*} P<0.05 ; t\right.$ test)

[Colour figure can be viewed at

wileyonlinelibrary.com]

oxidative damage under Al stress; hence, we used the fluorescence of H2DCF-DA as a read-out for the accumulation of $\mathrm{H}_{2} \mathrm{O}_{2}$ in roots. The results revealed no significant difference between WT/DJ, osaux3-2, and 35s:OsAUX3 PRs in the absence of Al, whereas fluorescence was increased in WT/DJ and 35S:OsAUX3 under 25- $\mu \mathrm{M}$ Al stress; however, there was no significant increase in osaux3-2 (Figure 7a). Interestingly, the fluorescence increased in 35S:OsAUX3-3 under Al stress was much more drastic than in WT/DJ. These results are consistent with a quantitative analysis of $\mathrm{H}_{2} \mathrm{O}_{2}$ concentration (Figure $7 \mathrm{~b}$ ), indicating that $\mathrm{Al}$ treatment increases the accumulation of $\mathrm{H}_{2} \mathrm{O}_{2}$ in the root apex of WT/DJ, osaux 3-2 and 35s:OsAUX3-3. However, the lower Al accumulation in osaux3-2 compared with the WT/DJ can reduce oxidative damage to PR apex in the presence of Al. On the other hand, expression of hydrogen peroxidase(CAT) in osaux3-2 was significantly higher than that of WT/DJ 


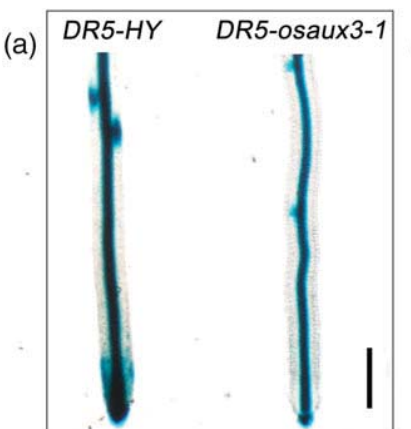

(c)

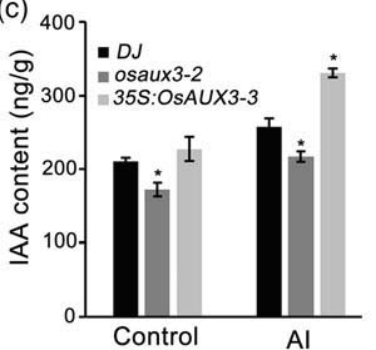

(d)
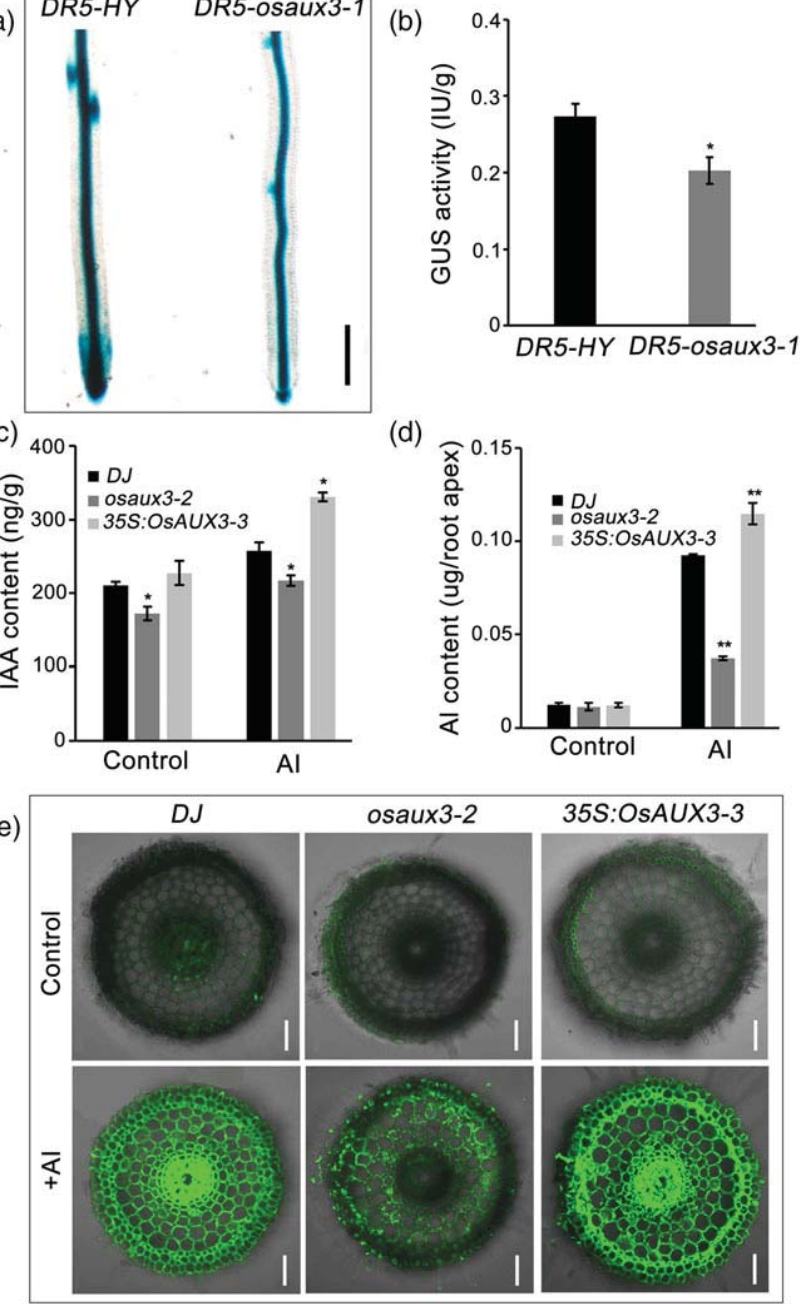

FIGURE 6 Auxin content and aluminium (Al) content in wild-type rice Dongjin (WT/DJ), osaux3-2, and 35s:OsAUX3-3 under Al stress. (a) $\beta$-Galactosidase (GUS) staining in primary root (PR) of ProDR5:GUS and osaux3-1 seedlings for 3-day-old. Ten biological replicas were analysed. Bar $=250 \mu \mathrm{m}$. (b) Quantification of ProDR5:GUS activity in the lines shown in (a). Five biological replicas were analysed in this test. Asterisks indicate significant differences to DR5-HY $\left({ }^{*} P<0.05 ; t\right.$ test). (c) Measurement of auxin concentrations in PR apex $(1 \mathrm{~cm})$ of 3 day-old WT/DJ, osaux3-2, and 35s:OsAUX3-3 seedlings under control and $25-\mu \mathrm{M} \mathrm{AlCl}{ }_{3}$ treatments. Five biological replicas were analysed in this test. Asterisks indicate significant differences compared with WT/ $D J$, respectively $\left({ }^{*} P<0.05\right.$; $t$ test). (d) Al content of PR apex $(1 \mathrm{~cm})$ of 3-day-old WT/DJ, osaux3-2, and 35S:OsAUX3-3 seedlings under Al stress. Five biological replicas were analysed in this test. Asterisks indicate significant differences compared with WT/DJ, respectively $\left({ }^{* *} P<0.01 ; t\right.$ test). (e) Morin staining of transverse section of PR apex $(1 \mathrm{~cm})$ of 3-day-old WT/DJ, osaux3-2, and 35s:OsAUX3-3 seedlings under Al stress. Bar $=50 \mu \mathrm{m}$. IAA: indole-3-acetic acid [Colour figure can be viewed at wileyonlinelibrary.com]

and 35S:OsAUX3-3, suggesting that more $\mathrm{H}_{2} \mathrm{O}_{2}$ was reduced to $\mathrm{H}_{2} \mathrm{O}$ and $\mathrm{O}_{2}$ under Al stress (Figure 7c).

\section{8 | Al-resistant genes are up-regulated in osaux3-2}

In rice, some genes have been reported to be involved in resistance to Al stress. Al RESISTANCE TRANSCRIPTION FACTOR1 (ART1), a transcription factor, encodes for a putative $\mathrm{C} 2 \mathrm{H} 2$ zinc finger protein, which is involved in Al tolerance by regulating multiple Al-tolerant genes (Yamaji et al., 2009). The ART1-regulated downstream genes, Nramp Al transporter1 (OsNrat1), rice ALUMINIUM SENSITIVE1 (OsALS1), and MAGNESIUM TRANSPORTER1 (OsMGT1), have been reported (Tsutsui, Yamaji, \& Feng, 2011). Nrat1 is localized to the plasma membranes of root tip cells and transports trivalent $\mathrm{Al}$ ions for Al detoxification (Xia, Yamaji, Kasai, \& Ma, 2010). OsALS1 is localized to the tonoplast, which is required for detoxification of $\mathrm{Al}$ in rice through sequestration of $\mathrm{Al}$ into vacuoles (Huang, Yamaji, Chen, \& Ma, 2012). OsMGT1, a putative rice Mg transporter, is able to alleviate $\mathrm{Al}$ toxicity through up-regulation of $\mathrm{Mg}$ concentrations under Al stress by displacing or competing with $\mathrm{Al}$ from binding sites of different cellular components (Chen, Yamaji, Motoyama, Nagamura, \& Ma, 2012).

To clarify how OsAUX3 responds to Al stress, we further analysed expression levels of these genes related to Al tolerance in WT/DJ, osaux3-2, and 35s:OsAUX3-3 under Al stress by qRT-PCR (Figure 7 d). The results reveal that the expression of these genes in the osaux 3-2 was higher than in WT/DJ and that their expression was dramatically up-regulated in osaux3-2 after Al treatment, whereas we found no significant increase in the 35S:OsAUX3-3 compared with the WT/DJ. This suggests that significant up-regulation of these Alresistant genes in osaux3-2 confers insensitivity to Al in osaux3-2.

\section{4 | DISCUSSION}

In the recent years, a participation of IAA in Al resistance of plants has frequently been reported. Inversely, Al has also been shown to affect root growth by modifying the levels of auxin (Ponce, Barlow, Feldman, \& Cassab, 2005); however, this study only presented indirect evidence on the involvement of polar transportation of auxin in Al-induced inhibition of root growth. The underlying molecular mechanisms of the effects of Al stress on auxin transport in rice are still unclear. Here, we uncover OsAUX3 as an auxin influx carrier, functioning in the regulation of root and its implication in responses to Al stress.

\section{1 | Unlike OsAUX1, OsAUX3 positively regulates PR growth}

In our previous report, OsAUX1 was shown to negatively regulate PR elongation (Yu et al., 2015). We therefore wondered if the role of OsAUX3 in PR development is similar to OsAUX1. According to sequence analysis of amino acids, a special domain (Figure S2c, with red box) in OsAUX3 is different to other OsAUX family members, including OsAUX1. Therefore, we used the CRISPR-Cas9 method to remove this special domain for constructing both osaux 3 mutants. As shown in Figure 1, both osaux 3 mutants revealed a short-root phenotype different to osaux1 (Yu et al., 2015). Therefore, this special domain in OsAUX3 might play an important role in PR development. In this study, we find short PR traits in osaux 3 mutants and longer PR in OsAUX3 overexpression lines that are opposite to the phenotypes of OsAUX1-related mutants and overexpression lines, respectively (Figure 1). Knock-down of OsAUX3 led to decreases in PR 

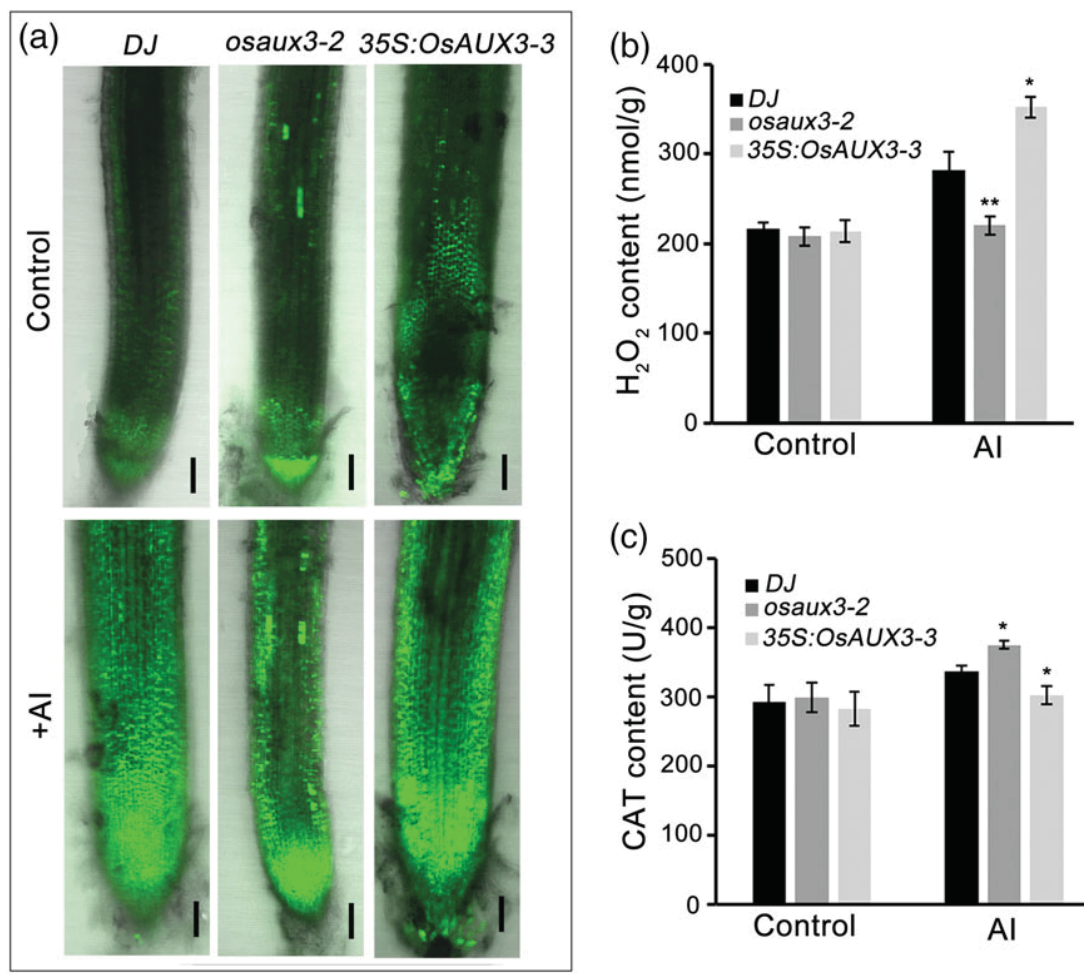

FIGURE $7 \quad \mathrm{H}_{2} \mathrm{O}_{2}$ accumulation under aluminium (Al) stress in wild-type rice Dongjin (WT/DJ), osaux3-2, and 35s:OsAUX3-3 and

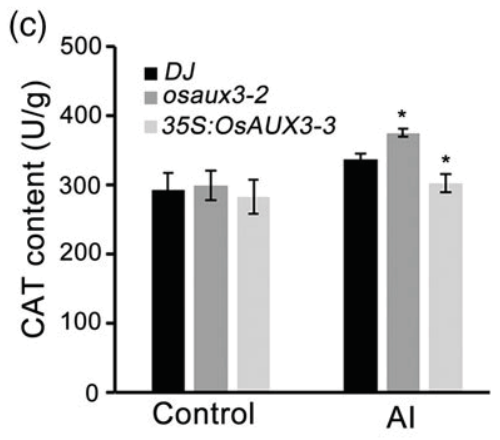
expression level of Al-resistant genes in primary root (PR) apex. (a) 2',7'-

Dichlorofluorescin diacetate staining (green) of PR apex $(1 \mathrm{~cm})$ in 3-day-old WT/DJ, osaux3-2, and 35S:OsAUX3-3 seedlings after Al treatment. Bar $=100 \mu \mathrm{m}$. (b) $\mathrm{H}_{2} \mathrm{O}_{2}$ content of PR apex $(1 \mathrm{~cm})$ in 3-day-old WT/DJ, osaux3-2, and 35S:OsAUX3-3 seedlings after Al stress. The experiment was analysed using five independent biological replicas. Asterisks indicate significant differences compared with WT/DJ, respectively $\left({ }^{* *} P<0.01 ;{ }^{*} P<0.05 ; t\right.$ test). (c) Catalase content of PR apex $(1 \mathrm{~cm})$ in 3-day-old WT/DJ, osaux3-2, and 35S: OsAUX3-3 seedlings after Al treatment. The experiment was analysed using five independent biological replicas. Asterisks indicate significant differences compared with WT/DJ, respectively $\left({ }^{*} P<0.05 ; t\right.$ test). (d) Relative mRNA level of Al-resistant genes in PR apex of 3-day-old WT/DJ, osaux3-2, and 35s:OsAUX3-3 seedling after Al treatment. OsACTIN and OsUBI were used as internal control. Columns with different letters indicate significant differences to control (Duncan's test, $P<0.05$ ) [Colour figure can be viewed at wileyonlinelibrary.com]

length, which suggests that OsAUX3 is also implicated in PR growth but positively regulates PR elongation. The expression of OsAUX3 in PR was induced by auxin, and shorter roots of osaux3-2 were insensitive to auxin, which is a significant auxin defective phenotype. EdU fluorescence staining showed a decrease of meristem cell activity in the PR of osaux3-2, indicating that inhibition of meristem cell division in PRs leads to reduced PR elongation. In agreement, the auxin distribution and concentration in osaux 3 was significantly decreased (Figure $6 \mathrm{a}-\mathrm{c}$ ), which demonstrates that OsAUX3 positively regulates PR growth by maintaining the auxin distribution in PRs to support meristem cell division required for normal PR growth.

\subsection{OsAUX3 controls LR initiation in analogy to AtAUX1, AtLAX3, and OsAUX1}

LR formation includes two phases: initiation and emergence (Bhalerao et al., 2002; Laskowski, Williams, Nusbaum, \& Sussex, 1995; Marchant et al., 2002). It was reported that AtAUX1 participates in the control of LR initiation and thus causes a reduction of LR number (Marchant et al., 1999; Marchant et al., 2002; Swarup et al., 2008). In Arabidopsis, the AUX1 and LIKE-AUXIN3 (LAX3) auxin influx carriers are required for auxin signalling activating LBD16 and LBD18 (lateral organ boundaries domain [LBD]) to control LR development (Lee, Cho, \& Kim, 2015). In rice, the osaux1 mutant has a reduced number of LR primordia (Zhao et al., 2015). In osaux3-2, all these feature including delayed LR initiation, decreased LR primordia, reduced LR density, and decreased expression of genes related to $L R$ initiation suggest that OsAUX3 plays an important role during LR initiation, which would be consistent with a conserved function to its homologues/analogues, AtAUX1, AtLAX3, or OsAUX1 (Figure 2).

\subsection{OsAUX3 regulates RH development different than AtAUX1 but similar to OsAUX1}

In Arabidopsis, the root epidermis consists of two cell types: one is called an RH cell forming RHs, and the other one is a non-hair cell, which does not form RHs. AtAUX1 is localized in non-hair epidermal cells, whereas OsAUX1 is expressed in young RH cells (Jones et al., 
2009; Yu et al., 2015). This means that AtAUX1 sustains RH development through regulating auxin transport in non-hair cells, whereas OsAUX1 directly regulates $\mathrm{RH}$, at least in young $\mathrm{RH}$ cells. Here, we show that OsAUX3 is expressed in either young or mature RH cells (Figure 3c), indicating that regulation of OsAUX3-mediated RH development is similar to OsAUX1 but different to AtAUX1 and that OsAUX3 might have a stronger biological function in regulating mature RH development than OsAUX1. Previous reports revealed that Arabidopsis belongs to the striped pattern (type 3) of $\mathrm{RHs}$, whereas rice was suggested to be type 2 , which depends on asymmetrical cell divisions (Clowes, 2000; Kwak \& Schiefelbein, 2007; Horn et al., 2009). In this study, the expression position of OsAUX3 further supports that rice $\mathrm{RHs}$ belong to type 2 .

\subsection{OsAUX3 is an auxin influx carrier playing an important role in acropetal auxin transport}

AtAUX1 is an influx carrier functioning in auxin uptake (Carrier et al., 2008; Dharmasiri et al., 2006; Peret et al., 2012; Robert \& Friml, 2009; Zazimalova, Murphy, Yang,Hoyerova, \& Hosek, 2010). LAX3 has been shown to create cell-specific auxin sinks (Swarup et al., 2008; Vandenbussche et al., 2010). Like AUX1 or LAX3 in Arabidopsis and OsAUX1 in rice, also, OsAUX3 is localized to plasma membrane (Figure 4a). Further, our study revealed reduced IAA export from osaux 3-1 and osaux3-2 protoplasts, indicating that OsAUX3 functions also as auxin influx carrier (Figure 4b). Acropetal auxin transport of osaux3-1 and osaux3-2 roots was found to be decreased in comparison with their WTs (Figure 4c,d), suggesting that OsAUX3 functions in acropetal transport of auxin. Decreased acropetal auxin transport results in a reduced auxin distribution and concentration in the PR apex of osaux3 (Figure 6a-c).

\subsection{OsAUX3 is induced in the root apex under Al stress and is involved in Al-induced inhibition of root growth}

Several studies have demonstrated that Al may interact with auxinsignalling pathways, leading to alterations of auxin accumulation and distribution in roots (Doncheva, Amenos, Poschenrieder, \& Barcelo, 2005; Kollmeier et al., 2000). In response to Al stress, auxin signalling in the root transition zone is enhanced, which results in auxin-regulated root growth inhibition through altering auxin response factors in Arabidopsis (Yang et al., 2014). In barley, auxin enhances Al-induced root growth inhibition in response to toxic Al stress (Bai et al., 2017). In wheat, MAPK-mediated auxin signal transduction pathways triggers an increase of malic acid against Al toxicity (Liu et al., 2017).

Rice is one of the most Al-tolerant species. Our results demonstrate that the presence of Al promotes the accumulation of auxin in the root apex of rice. However, in osaux3-2, the increase of auxin contents was less pronounced than in WT/DJ (Figure 6c). Hence, root growth inhibition under Al stress was also significantly alleviated in osaux3-2 compared with WT/DJ (Figure 5c-h), uncovering that OsAUX3 plays an important role in regulating the auxin content of the root apex in the presence of Al. On the other hand, auxin negatively regulates $\mathrm{Al}$ tolerance through altering ALS1 expression and Al distribution within Arabidopsis cells (Zhu et al., 2013). Al suppresses root growth due to an abnormal accumulation of auxin and cytokinin in Arabidopsis (Daspute et al., 2017). In agreement, our experiments show that $\mathrm{Al}$ accumulation in the PR apex of osaux3-2 was reduced compared with WT/DJ (Figure 6d,e). This confirms that auxin negatively regulates $\mathrm{Al}$ tolerance in rice and that this process involves OsAUX3-mediated auxin transport.

\subsection{Reduced oxidative damage in the PR of osaux3- 2 under Al stress is regulated through enhanced expression of Al-tolerance-related genes}

Auxin was suggested to regulate ROS level and to direct the role of ROS in oxidative stress (Iglesias, Terrile, Bartoli, D'Ippolito, \& Casalongue, 2010; Krishnamurthy \& Rathinasabapathi, 2013b). Auxin plays an important role in responses to oxidative stress and effects the distribution of Al in cells (Krishnamurthy \& Rathinasabapathi, 2013a; Zhu et al., 2013). Phytohormones and ROS activate various transcriptional responses, including the expression of genes related to increased Al tolerance under Al treatment (Daspute et al., 2017). Our results show that loss of OsAUX3-mediated acropetal auxin transport causing decreased Al distribution in root cells resulted in distinctly alleviated Al-induced oxidative cellular damage in the rice root apex of osaux3-2 (Figures 4 and 7). We uncover a novel pathway that employs inhibition of auxin acropetal transport to alleviate Al-induced oxidative cellular damage. Our findings offer a novel path for engineering Al-tolerance rice species by altering the expression of the auxin influx carrier OsAUX3.

Previous studies identified some Al-tolerance-related genes in rice, including OsART1, OsNrat1, OsALS1, and OsMGT1, whose expression was induced by Al (Chen et al., 2012; Huang et al., 2012; Xia et al., 2010; Yamaji et al., 2009). Our results further show that expression of these Al-tolerance-related genes in osaux3-2 was more upregulated than in WT/DJ upon Al treatment. As previously reported, OsNrat1 and OsALS1 play important role in detoxifying Al through transportation and sequestration of $\mathrm{Al}$ into vacuoles (Huang et al., 2012; Xia et al., 2010). The up-regulation of these Al-tolerance-related genes might result in the reduced oxidative damage to the PR apex in osaux3-2 in the presence of Al.

Taken together, our results indicate that decreased auxin transport in osaux 3 mutants is responsible for its reduced sensitivity towards Al stress, underlining that auxin plays an important role as a signalling molecular in response to Al stress.

\section{ACKNOWLEDGEMENTS}

This project was funded by grants from the National Basic Research Program of China (973 Program, Grant 2015CB942900), the National Natural Science Foundation of China (Grant 31471460), the State Key Laboratory of Plant Genomics (2017A0407-13), 111 project (Grant B14027), and the Swiss National Funds (Grant 31003A_165877 to M. G.). We gratefully acknowledge Prof. YiNong Yang for contributing CRISPR/Cas9 system. 


\section{ORCID}

YanHua Qi (1) https://orcid.org/0000-0002-6234-5317

\section{REFERENCES}

Bai, B., Bian, H., Zeng, Z., Hou, N., Shi, B., Wang, J., ... Han, N. (2017). miR393-mediated auxin signaling regulation is involved in root elongation inhibition in response to toxic aluminum stress in barley. Plant \& Cell Physiology, 58, 426-439. https://doi.org/10.1093/pcp/pcw211

Barbez, E., \& Kleine-Vehn, J. (2013). Divide Et Impera--cellular auxin compartmentalization. Current Opinion in Plant Biology, 16, 78-84. https:// doi.org/10.1016/j.pbi.2012.10.005

Bennett, M. J., Marchant, A., Green, H. G., May, S. T., Ward, S. P., Millner, P. A., ... Feldmann, K. A. (1996). Arabidopsis AUX1 gene: A permease-like regulator of root gravitropism. Science, 273, 948-950. https://doi.org/ $10.1126 /$ science. 273.5277 .948

Bhalerao, R. P., Eklof, J., Ljung, K., Marchant, A., Bennett, M., \& Sandberg, G. (2002). Shoot-derived auxin is essential for early lateral root emergence in Arabidopsis seedlings. The Plant Journal, 29, 325-332. https://doi.org/10.1046/j.0960-7412.2001.01217.x

Bhosale, R., Giri, J., Pandey, B. K., Giehl, R., Hartmann, A., Traini, R., ... Swarup, R. (2018). A mechanistic framework for auxin dependent Arabidopsis root hair elongation to low external phosphate. Nature Communications, 9, 1409. https://doi.org/10.1038/s41467-018-03851-3

Carrier, D. J., Bakar, N. T., Swarup, R., Callaghan, R., Napier, R. M., Bennett, M. J., \& Kerr, I. D. (2008). The binding of auxin to the Arabidopsis auxin influx transporter AUX1. Plant Physiology, 148, 529-535. https://doi. org/10.1104/pp.108.122044

Chen, Z. C., Yamaji, N., Motoyama, R., Nagamura, Y., \& Ma, J. F. (2012). Upregulation of a magnesium transporter gene OsMGT1 is required for conferring aluminum tolerance in rice. Plant Physiology, 159, 1624-1633. https://doi.org/10.1104/pp.112.199778

Cho, M., Lee, S. H., \& Cho, H. T. (2007). P-glycoprotein4 displays auxin efflux transporter-like action in Arabidopsis root hair cells and tobacco cells. Plant Cell, 19, 3930-3943. https://doi.org/10.1105/tpc.107.054288

Clowes, F. A. L. (2000). Pattern in root meristem development in angiosperms. The New Phytologist, 146, 83-94. https://doi.org/10.1046/ j.1469-8137.2000.00614.x

Daspute, A. A., Sadhukhan, A., Tokizawa, M., Kobayashi, Y., Panda, S. K., \& Koyama, H. (2017). Transcriptional regulation of aluminum-tolerance genes in higher plants: Clarifying the underlying molecular mechanisms. Frontiers in Plant Science, 8, 1358. https://doi.org/10.3389/ fpls.2017.01358

Del, C. P., Llorente, A. R., Gomez, A., Gosalvez, J., Goyanes, V. J., \& Stockert, J. C. (1990). New fluorescence reactions in DNA cytochemistry. 2. Microscopic and spectroscopic studies on fluorescent aluminum complexes. Analytical and Quantitative Cytology and Histology, 12, 11-20.

Delhaize, E., \& Ryan, P. R. (1995). Aluminum toxicity and tolerance in plants. Plant Physiology, 107, 315-321. https://doi.org/10.1104/ pp.107.2.315

Dharmasiri, S., Swarup, R., Mockaitis, K., Dharmasiri, N., Singh, S. K., Kowalchyk, M., ... Estelle, M. (2006). AXR4 is required for localization of the auxin influx facilitator AUX1. Science, 312, 1218-1220. https://doi.org/10.1126/science.1122847

Doncheva, S., Amenos, M., Poschenrieder, C., \& Barcelo, J. (2005). Root cell patterning: A primary target for aluminium toxicity in maize. Journal of Experimental Botany, 56, 1213-1220. https://doi.org/10.1093/jxb/ eri115

Foy, C. D. (1988). Plant adaptation to acid, aluminum-toxic soils. Communications in Soil Science and Plant Analysis, 19, 959-987. https://doi.org/ 10.1080/00103628809367988

Friml, J., \& Palme, K. (2002). Polar auxin transport-Old questions and new concepts? Plant Molecular Biology, 49, 273-284. https://doi.org/ 10.1023/A:1015248926412
Gao, L. W., Lyu, S. W., Tang, J., Zhou, D. Y., Bonnema, G., Xiao, D., ... Zhang, C. W. (2017). Genome-wide analysis of auxin transport genes identifies the hormone responsive patterns associated with leafy head formation in Chinese cabbage. Scientific Reports, 7, 42229. https://doi. org/10.1038/srep42229

Geisler, M., Blakeslee, J. J., Bouchard, R., Lee, O. R., Vincenzetti, V., Bandyopadhyay, A., ... Martinoia, E. (2005). Cellular efflux of auxin catalyzed by the Arabidopsis MDR/PGP transporter AtPGP1. The Plant Journal, 44, 179-194. https://doi.org/10.1111/j.1365313X.2005.02519.x

Guo, J., Song, J., Wang, F., \& Zhang, X. S. (2007). Genome-wide identification and expression analysis of rice cell cycle genes. Plant Molecular Biology, 64, 349-360. https://doi.org/10.1007/s11103-007-9154-y

Hiei, Y., Ohta, S., Komari, T., \& Kumashiro, T. (1994). Efficient transformation of rice (Oryza sativa L.) mediated by Agrobacterium and sequence analysis of the boundaries of the T-DNA. The Plant Journal, 6, 271-282. https://doi.org/10.1046/j.1365-313X.1994.6020271.x

Horn, R., Yi, K., Menand, B., Pernas-Ochoa, M., Takeda, S., Walker, T., \& Dolan, L. (2009). Root epidermal development in Arabidopsis. Annual Plant Reviews, 37, 64-82.

Huang, C. F., Yamaji, N., Chen, Z., \& Ma, J. F. (2012). A tonoplast-localized half-size $A B C$ transporter is required for internal detoxification of aluminum in rice. The Plant Journal, 69, 857-867. https://doi.org/ 10.1111/j.1365-313X.2011.04837.x

Iglesias, M. J., Terrile, M. C., Bartoli, C. G., D'Ippolito, S., \& Casalongue, C. A. (2010). Auxin signaling participates in the adaptative response against oxidative stress and salinity by interacting with redox metabolism in Arabidopsis. Plant Molecular Biology, 74, 215-222. https://doi. org/10.1007/s11103-010-9667-7

Jefferson, R. A., Kavanagh, T. A., \& Bevan, M. W. (1987). GUS fusions: Beta-glucuronidase as a sensitive and versatile gene fusion marker in higher plants. The EMBO Journal, 6, 3901-3907. https://doi.org/ 10.1002/j.1460-2075.1987.tb02730.x

Jones, A. R., Kramer, E. M., Knox, K., Swarup, R., Bennett, M. J., Lazarus, C. M., ... Grierson, C. S. (2009). Auxin transport through non-hair cells sustains root-hair development. Nature Cell Biology, 11, 78-84. https:// doi.org/10.1038/ncb1815

Kepinski, S. (2007). The anatomy of auxin perception. BioEssays, 29, 953-956. https://doi.org/10.1002/bies.20657

Kerr, I. D., \& Bennett, M. J. (2007). New insight into the biochemical mechanisms regulating auxin transport in plants. The Biochemical Journal, 401, 613-622. https://doi.org/10.1042/BJ20061411

Kochian, L. V. (1995). Cellular mechanisms of aluminum toxicity and resistance in plants. Annual Review of Plant Physiology and Plant Molecular Biology, 46, 237-260. https://doi.org/10.1146/annurev. pp.46.060195.001321

Kollmeier, M., Felle, H. H., \& Horst, W. J. (2000). Genotypical differences in aluminum resistance of maize are expressed in the distal part of the transition zone. Is reduced basipetal auxin flow involved in inhibition of root elongation by aluminum? Plant Physiology, 122, 945-956. https://doi.org/10.1104/pp.122.3.945

Kramer, E. M., \& Bennett, M. J. (2006). Auxin transport: A field in flux Trends in Plant Science, 11, 382-386. https://doi.org/10.1016/j. tplants.2006.06.002

Krishnamurthy, A., \& Rathinasabapathi, B. (2013a). Auxin and its transport play a role in plant tolerance to arsenite-induced oxidative stress in Arabidopsis thaliana. Plant, Cell \& Environment, 36, 1838-1849. https://doi.org/10.1111/pce.12093

Krishnamurthy, A., \& Rathinasabapathi, B. (2013b). Oxidative stress tolerance in plants: Novel interplay between auxin and reactive oxygen species signaling. Plant Signaling \& Behavior, 8, 10-4161.

Kwak, S. H., \& Schiefelbein, J. (2007). The role of the SCRAMBLED receptorlike kinase in patterning the Arabidopsis root epidermis. Developmental Biology, 302, 118-131. https://doi.org/10.1016/j.ydbio.2006.09.009 
Laskowski, M. J., Williams, M. E., Nusbaum, H. C., \& Sussex, I. M. (1995). Formation of lateral root meristems is a two-stage process. Development, 121, 3303-3310.

Lee, H. W., Cho, C., \& Kim, J. (2015). Lateral organ boundaries domain16 and 18 Act downstream of the AUXIN1 and LIKE-AUXIN3 auxin influx carriers to control lateral root development in Arabidopsis. Plant Physiology, 168, 1792-1806. https://doi.org/10.1104/pp.15.00578

Liu, X., Lin, Y., Liu, D., Wang, C., Zhao, Z., Cui, X., ... Yang, Y. (2017). MAPKmediated auxin signal transduction pathways regulate the malic acid secretion under aluminum stress in wheat (Triticum aestivum L.). Scientific Reports, 7, 1620.

Ljung, K. (2013). Auxin metabolism and homeostasis during plant development. Development, 140, 943-950. https://doi.org/10.1242/ dev.086363

Marchant, A., Bhalerao, R., Casimiro, I., Eklof, J., Casero, P. J., Bennett, M., \& Sandberg, G. (2002). AUX1 promotes lateral root formation by facilitating indole-3-acetic acid distribution between sink and source tissues in the Arabidopsis seedling. Plant Cell, 14, 589-597. https:// doi.org/10.1105/tpc.010354

Marchant, A., Kargul, J., May, S. T., Muller, P., Delbarre, A., PerrotRechenmann, C., \& Bennett, M. J. (1999). AUX1 regulates root gravitropism in Arabidopsis by facilitating auxin uptake within root apical tissues. The EMBO Journal, 18, 2066-2073. https://doi.org/ 10.1093/emboj/18.8.2066

Mile, I., Svensson, A., Darabi, A., Mold, M., Siesjo, P., \& Eriksson, H. (2015). Al adjuvants can be tracked in viable cells by lumogallion staining. Journal of Immunological Methods, 422, 87-94. https://doi.org/10.1016/j. jim.2015.04.008

Murphy, A. S., Hoogner, K. R., Peer, W. A., \& Taiz, L. (2002). Identification, purification, and molecular cloning of $\mathrm{N}$-1-naphthylphthalmic acidbinding plasma membrane-associated aminopeptidases from Arabidopsis. Plant Physiology, 128, 935-950. https://doi.org/ 10.1104/pp.010519

Nieuwland, J., Maughan, S., Dewitte, W., Scofield, S., Sanz, L., \& Murray, J. A. (2009). The D-type cyclin CYCD4;1 modulates lateral root density in Arabidopsis by affecting the basal meristem region. Proceedings of the National Academy of Sciences of the United States of America, 106, 22528-22533. https://doi.org/10.1073/pnas.0906354106

Noh, B., Murphy, A. S., \& Spalding, E. P. (2001). Multidrug resistance-like genes of Arabidopsis required for auxin transport and auxin-mediated development. Plant Cell, 13, 2441-2454. https://doi.org/10.1105/ tpc.13.11.2441

Pattison, R. J., \& Catala, C. (2012). Evaluating auxin distribution in tomato (Solanum lycopersicum) through an analysis of the PIN and AUX/LAX gene families. The Plant Journal, 70, 585-598. https://doi.org/ 10.1111/j.1365-313X.2011.04895.x

Peret, B., Swarup, K., Ferguson, A., Seth, M., Yang, Y., Dhondt, S., ... Swarup, R. (2012). AUX/LAX genes encode a family of auxin influx transporters that perform distinct functions during Arabidopsis development. Plant Cell, 24, 2874-2885. https://doi.org/10.1105/ tpc.112.097766

Petrasek, J., \& Friml, J. (2009). Auxin transport routes in plant development. Development, 136, 2675-2688. https://doi.org/10.1242/dev.030353

Petrasek, J., Mravec, J., Bouchard, R., Blakeslee, J. J., Abas, M., Seifertova, D., ... Friml, J. (2006). PIN proteins perform a rate-limiting function in cellular auxin efflux. Science, 312, 914-918. https://doi.org/10.1126/ science.1123542

Ponce, G., Barlow, P. W., Feldman, L. J., \& Cassab, G. I. (2005). Auxin and ethylene interactions control mitotic activity of the quiescent centre, root cap size, and pattern of cap cell differentiation in maize. Plant, Cell \& Environment, 28, 719-732. https://doi.org/10.1111/j.1365-3040.2005.01318.x

Qi, J., Qian, Q., Bu, Q., Li, S., Chen, Q., Sun, J., ... Li, C. (2008). Mutation of the rice Narrow leaf1 gene, which encodes a novel protein, affects vein patterning and polar auxin transport. Plant Physiology, 147, 1947-1959. https://doi.org/10.1104/pp.108.118778
Qi, Y., Wang, S., Shen, C., Zhang, S., Chen, Y., Xu, Y., ... Jiang, D. (2012). OsARF12, a transcription activator on auxin response gene, regulates root elongation and affects iron accumulation in rice (Oryza sativa). The New Phytologist, 193, 109-120. https://doi.org/10.1111/j.14698137.2011.03910.x

Robert, H. S., \& Friml, J. (2009). Auxin and other signals on the move in plants. Nature Chemical Biology, 5, 325-332. https://doi.org/10.1038/ nchembio.170

Roy, S., Robson, F., Lilley, J., Liu, C. W., Cheng, X., Wen, J., ... Murray, J. D. (2017). MtLAX2, a functional homologue of the Arabidopsis auxin influx transporter AUX1, is required for nodule organogenesis. Plant Physiology, 174, 326-338. https://doi.org/10.1104/pp.16.01473

Sanz, L., Dewitte, W., Forzani, C., Patell, F., Nieuwland, J., Wen, B., ... Murray, J. A. (2011). The Arabidopsis D-type cyclin CYCD2;1 and the inhibitor ICK2/KRP2 modulate auxin-induced lateral root formation. Plant Cell, 23, 641-660. https://doi.org/10.1105/tpc.110.080002

Shen, C., Bai, Y., Wang, S., Zhang, S., Wu, Y., Chen, M., ... Qi, Y. (2010). Expression profile of PIN, AUX/LAX and PGP auxin transporter gene families in Sorghum bicolor under phytohormone and abiotic stress. The FEBS Journal, 277, 2954-2969. https://doi.org/10.1111/j.17424658.2010.07706.x

Swarup, K., Benkova, E., Swarup, R., Casimiro, I., Peret, B., Yang, Y., ... Bennett, M. J. (2008). The auxin influx carrier LAX3 promotes lateral root emergence. Nature Cell Biology, 10, 946-954. https://doi.org/ 10.1038/ncb1754

Swarup, R., \& Bennett, M. (2003). Auxin transport: The fountain of life in plants? Developmental Cell, 5, 824-826. https://doi.org/10.1016/ S1534-5807(03)00370-8

Swarup, R., Friml, J., Marchant, A., Ljung, K., Sandberg, G., Palme, K., \& Bennett, M. (2001). Localization of the auxin permease AUX1 suggests two functionally distinct hormone transport pathways operate in the Arabidopsis root apex. Genes \& Development, 15, 2648-2653. https:// doi.org/10.1101/gad.210501

Teale, W. D., Paponov, I. A., \& Palme, K. (2006). Auxin in action: Signalling, transport and the control of plant growth and development. Nature Reviews. Molecular Cell Biology, 7, 847-859. https://doi.org/10.1038/ nrm2020

Tsutsui, T., Yamaji, N., \& Feng, M. J. (2011). Identification of a cis-acting element of ART1, a C2H2-type zinc-finger transcription factor for aluminum tolerance in rice. Plant Physiology, 156, 925-931. https://doi. org/10.1104/pp.111.175802

von Uexküll, H. R., \& Mutert, E. (1995). Global extent, development and economic impact of acid soils. In R. A. Date, N. J. Grundon, G. E. Raymet, \& M. E. Probert (Eds.), Plant-soil interactions at low pH: Principles and management (pp. 5-19). Dordrecht, The Netherlands: Kluwer Academic Publishers. https://doi.org/10.1007/978-94-011-0221-6_1

Vandenbussche, F., Petrasek, J., Zadnikova, P., Hoyerova, K., Pesek, B., Raz, V., ... Van Der Straeten, D. (2010). The auxin influx carriers AUX1 and LAX3 are involved in auxin-ethylene interactions during apical hook development in Arabidopsis thaliana seedlings. Development, 137, 597-606. https://doi.org/10.1242/dev.040790

Verkest, A., Manes, C. L., Vercruysse, S., Maes, S., Van Der Schueren, E., Beeckman, T., ... De Veylder, L. (2005). The cyclin-dependent kinase inhibitor KRP2 controls the onset of the endoreduplication cycle during Arabidopsis leaf development through inhibition of mitotic CDKA;1 kinase complexes. Plant Cell, 17, 1723-1736. https://doi.org/ 10.1105/tpc.105.032383

Vieten, A., Sauer, M., Brewer, P. B., \& Friml, J. (2007). Molecular and cellular aspects of auxin-transport-mediated development. Trends in Plant Science, 12, 160-168. https://doi.org/10.1016/j.tplants.2007.03.006

Wang, R., Wang, J., Zhao, L., Yang, S., \& Song, Y. (2015). Impact of heavy metal stresses on the growth and auxin homeostasis of Arabidopsis seedlings. Biometals, 28, 123-132. https://doi.org/10.1007/s10534014-9808-6

Wang, S., Bai, Y., Shen, C., Wu, Y., Zhang, S., Jiang, D., ... Qi, Y. (2010). Auxin-related gene families in abiotic stress response in Sorghum 
bicolor. Functional \& Integrative Genomics, 10, 533-546. https://doi. org/10.1007/s10142-010-0174-3

Wang, S., Xu, Y., Li, Z., Zhang, S., Lim, J. M., Lee, K. O., ... Qi, Y. (2014). OsMOGS is required for $\mathrm{N}$-glycan formation and auxin-mediated root development in rice (Oryza sativa L.). The Plant Journal, 78, 632-645. https://doi.org/10.1111/tpj.12497

Wu, D., Shen, H., Yokawa, K., \& Baluska, F. (2014). Alleviation of aluminium-induced cell rigidity by overexpression of OsPIN2 in rice roots. Journal of Experimental Botany, 65, 5305-5315. https://doi.org/ 10.1093/jxb/eru292

Xia, J., Yamaji, N., Kasai, T., \& Ma, J. F. (2010). Plasma membrane-localized transporter for aluminum in rice. Proceedings of the National Academy of Sciences of the United States of America, 107, 18381-18385. https:// doi.org/10.1073/pnas.1004949107

Xie, K., Minkenberg, B., \& Yang, Y. (2015). Boosting CRISPR/Cas9 multiplex editing capability with the endogenous tRNA-processing system. Proceedings of the National Academy of Sciences of the United States of America, 112, 3570-3575. https://doi.org/10.1073/ pnas.1420294112

Xu, Y., Zhang, S., Guo, H., Wang, S., Xu, L., Li, C., ... Jiang, D. A. (2014). OsABCB14 functions in auxin transport and iron homeostasis in rice (Oryza sativa L.). The Plant Journal, 79, 106-117. https://doi.org/ 10.1111/tpj.12544

Yamaji, N., Huang, C. F., Nagao, S., Yano, M., Sato, Y., Nagamura, Y., \& Ma, J. F. (2009). A zinc finger transcription factor ART1 regulates multiple genes implicated in aluminum tolerance in rice. Plant Cell, 21, 3339-3349. https://doi.org/10.1105/tpc.109.070771

Yamamoto, Y., Kobayashi, Y., Devi, S. R., Rikiishi, S., \& Matsumoto, H. (2002). Aluminum toxicity is associated with mitochondrial dysfunction and the production of reactive oxygen species in plant cells. Plant Physiology, 128, 63-72. https://doi.org/10.1104/pp.010417

Yang, H., \& Murphy, A. S. (2009). Functional expression and characterization of Arabidopsis ABCB, AUX 1 and PIN auxin transporters in Schizosaccharomyces pombe. The Plant Journal, 59, 179-191. https:// doi.org/10.1111/j.1365-313X.2009.03856.x

Yang, Z. B., Geng, X., He, C., Zhang, F., Wang, R., Horst, W. J., \& Ding, Z. (2014). TAA1-regulated local auxin biosynthesis in the root-apex transition zone mediates the aluminum-induced inhibition of root growth in
Arabidopsis. Plant Cell, 26, 2889-2904. https://doi.org/10.1105/ tpc.114.127993

Yang, Z. B., Liu, G., Liu, J., Zhang, B., Meng, W., Muller, B., ... Ding, Z (2017). Synergistic action of auxin and cytokinin mediates aluminuminduced root growth inhibition in Arabidopsis. EMBO Reports, 18, 1213-1230. https://doi.org/10.15252/embr.201643806

Yu, C., Sun, C., Shen, C., Wang, S., Liu, F., Liu, Y., ... Qi, Y. (2015). The auxin transporter, OsAUX1, is involved in primary root and root hair elongation and in $\mathrm{Cd}$ stress responses in rice (Oryza sativa L.). The Plant Journal, 83, 818-830. https://doi.org/10.1111/tpj.12929

Zazimalova, E., Murphy, A. S., Yang, H., Hoyerova, K., \& Hosek, P. (2010). Auxin transporters-Why so many? Cold Spring Harbor Perspectives in Biology, 2, a1552. https://doi.org/10.1101/cshperspect.a001552

Zhao, H., Ma, T., Wang, X., Deng, Y., Ma, H., Zhang, R., \& Zhao, J. (2015). OsAUX1 controls lateral root initiation in rice (Oryza sativa L.). Plant, Cell \& Environment, 38, 2208-2222. https://doi.org/10.1111/pce.12467

Zhou, P., Yang, F., Ren, X., Huang, B., \& An, Y. (2014). Phytotoxicity of aluminum on root growth and indole-3-acetic acid accumulation and transport in alfalfa roots. Environmental and Experimental Botany, 104 1-8. https://doi.org/10.1016/j.envexpbot.2014.02.018

Zhu, X. F., Lei, G. J., Wang, Z. W., Shi, Y. Z., Braam, J., Li, G. X., \& Zheng, S. J. (2013). Coordination between apoplastic and symplastic detoxification confers plant aluminum resistance. Plant Physiology, 162, 1947-1955. https://doi.org/10.1104/pp.113.219147

\section{SUPPORTING INFORMATION}

Additional supporting information may be found online in the Supporting Information section at the end of the article. 